Anomalous Reflections Near a. Caustic

\author{
Clifford J. Nolan \\ William W. Symes
}

July 1995

TR95-23 



\title{
Anomalous Reflections Near a Caustic
}

\author{
Clifford J. Nolan William W. Symes
}

May 19, 1995

\begin{abstract}
We consider scattering associated to the reduced scalar wave equation. High frequency asymptotic solutions of this equation leads to the theory of geometrical optics. In this theory energy is transported along rays (orthogonal trajectories to wavefronts). However this theory breaks down as soon as the ray field forms an envelope called a caustic. This signals that a dramatic change in nature of wave propagation occurs in the vicinity of a caustic. To illustrate this change of character we study an experiment which shows that reflected waves may have arbitrarily high energy content relative to the "size" of the scatterer. Moreover a theorem is proved showing that this unbounded behaviour can only occur when a caustic develops.
\end{abstract}

\section{Introduction}

Our motivation for this study is the scattering of acoustic waves in the subsurface of the earth. However the results are completely general, applying to any physical phenomenon described by the scalar wave equation. We shall set up two physical models to demonstrate applicability of our results. First we describe the scattering problem in geophysics and then an analagous problem in electromagnetism.

For simplicity we consider the earth as a two dimesnsional halfspace (although the results in this paper can be generaized to any dimension), with cartesian coordinates $(x, y)$ occupied by a fluid which has constant density and propagates pressure waves in its interior at a local speed $c(x, y)$. The Green's function $p(x, y, t)$ associated to pressure waves travelling in the subsurface of the earth as a result of an energy source with time signature $f \in L^{2}(\mathbf{R})$ being fired on the surface at location $\mathbf{x}_{s}$ satisfies the acoustic scalar wave equation

$$
c^{-2}(x, y) \frac{\partial^{2} p}{\partial t^{2}}(x, y, t)-\Delta p(x, y, t)=f(t) \delta\left(\mathbf{x}-\mathbf{x}_{s}\right)
$$

Similarly in the electromagnetic case we have an electromagnetic field propagating in a two dimensional half space as above. The electromagnetic field obeys Maxwell's equations. Let $\mathbf{D}, \mathbf{B}, \mathbf{E}, \mathbf{H}, \mathbf{J}$ respectively denote the displacement current, the magnetic field, the 
electric field, the magnetic intensity, and the current density. Then Maxwell's equations are

$$
\begin{array}{r}
\nabla \cdot \mathbf{D}=\rho_{\text {free }} \\
\nabla \cdot \mathbf{B}=0 \\
\dot{\nabla} \times \mathbf{E}=-\frac{\partial \mathbf{B}}{\partial t} \\
\nabla \times \mathbf{B}=\mathbf{J}_{\text {free }}+\frac{\partial \mathbf{D}}{\partial t} .
\end{array}
$$

In addition to these equations we have the constitutive equations

$$
\begin{array}{r}
\mathbf{J}=\sigma \mathbf{E} \\
\mathbf{B}=\left(\mu_{0} \mu\right)^{-1} \mathbf{H} \\
\mathbf{D}=\epsilon_{0} \epsilon \mathbf{E}
\end{array}
$$

where, $\epsilon$ is the relative electric permitivity tensor and $\epsilon_{0}$ is its value in free space. Similarly $\mu$ is the magnetic permeability tensor and $\mu_{0}$ is its value in free space.

Now we make some simplifications to get to the reduced wave equation for the electromagnetic field.

Assume that the electromagnetic field is studied in a 2 dimensional half space which is electrically neutral $\left(\rho_{\text {free }}=0\right)$, non-magnetic $\left(\mu=\mu_{0}\right)$, and non-conductive $(\sigma=0)$. Coordinates on this half space will be cartesian and a typical point will be denoted by $(x, y)$ and in future we will often write $\mathbf{x}$ in boldface for $(x, y)$.

With these assumptions, it then follows [7] from Maxwell's equations (1.2) that for an electric field polarized along the $x$-axis

$$
\mathbf{E}(x, y, t)=(\dot{\psi}(x, y, t), 0)
$$

satisfies the reduced wave equation equation

$$
\begin{array}{r}
c^{-2}(x, y) \frac{\partial^{2} \psi}{\partial t^{2}}(x, y, t)-\Delta \psi(x, y, t)=\frac{\partial}{\partial x}\left(\psi \frac{\partial \ln \epsilon}{\partial x}\right) \\
\text { where } 0<c(x, y) \equiv \sqrt{\frac{\epsilon_{0}(x, y) \mu_{0}(x, y)}{\epsilon(x, y)}} .
\end{array}
$$

At this stage we start our general study of the scalar wave equation. 
We study the related Green's function for the reduced wave equation

$$
c^{-2}(x, y) \frac{\partial^{2} G}{\partial t^{2}}(\mathbf{x}, t)-\Delta G(\mathbf{x}, t)=\delta(t) \delta\left(\mathbf{x}-\mathbf{x}_{\mathbf{s}}\right)
$$

corresponding to an impulsive source at location $\left(x_{s}, 0\right)$ - see figure $(1.1)$ for the corresponding experiment. This enabales us to find $\psi$, since $\psi$ is just the right hand side of (1.5)i convolved with $G$.

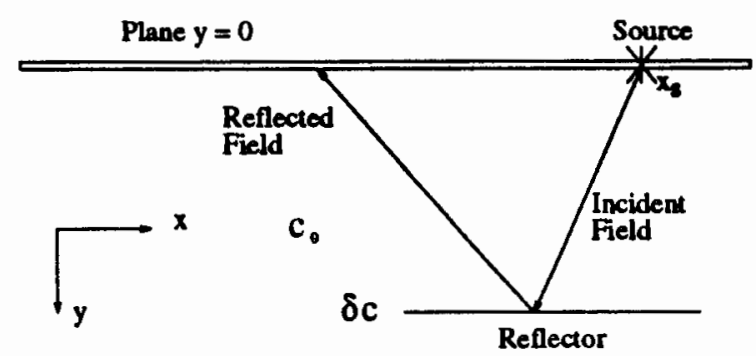

Figure 1.1: The experiment

Assume that the coefficient $c$ has been split into two pieces

$$
c(\mathbf{x})=c_{0}(\mathbf{x})+\delta c(\mathbf{x})
$$

where $c_{0}$ is a smooth function and $\delta c$ has much higher frequency content than $c_{0}$.

Denote by $G_{0}$ the solution of (1.6) with $c$ replaced by $c_{0}$. In (1.6), write $G=G_{0}+\delta G$ to define $\delta G$. Then if one linearizes equation (1.6) about $c_{0}, G_{0}$ one finds that $\delta G$ satisfies the linearized wave equation

$$
\frac{1}{c^{2}} \frac{\partial^{2} \delta G}{\partial t^{2}}-\nabla \cdot(\nabla \delta G)=\frac{2 \delta c}{c_{0}^{3}} \frac{\partial^{2} G_{0}}{\partial t^{2}}
$$

Thus $\delta c$ appears as a source for the (scattered) wavefield $\delta G$. Then $G_{0}+\delta G$ is the Born approximation to $G$.

Our ultimate goal is to solve the following inverse problem. Given that we record the scattered field on a finite portion $[0, L]$ of the line $y=0$, deduce $\delta c$. Crucial to the solving of such an inverse problem is a good understanding of the nature of the forward problem i.e. the operator which maps $\delta c$ into such a recording on $y=0$. We now formally define this operator and proceed to investigate its character in the later sections. We do not emphasize the inverse problem in this paper, but instead make reference to the implication of our results for it.

\section{Definition}

$$
(\mathcal{F} \delta c)(x, t)=m(x, t) \cdot \delta G(x, 0, t)
$$


where $m \in C_{0}^{\infty}\left(R_{x} \times[0, T]\right)$ is a cutoff function which is used to reflect the fact that we only record the scattered field for a finite time $T$ and for a finite portion $[0, L]$ of the line $y=0$.

Note that we are focussing on the linear behaviour of $\mathcal{F}$ as a function of the perturbation $\delta c$ alone. Thus, tacitly we assume that we have estimated $c_{0}$ (there are various techniques available for this ) in order to get an expression for $G_{0}$ (actually an asymptotic expansion for $G_{0}$ will be used) as we shall later see.

\section{The Main Result}

We will show that if we impose some mild restrictions on the geometry of rays travelling in the medium defined by the coefficient $c_{0}$, then we have that for a certain Hilbert space $\mathcal{H}$ that

$$
\mathcal{F}: \mathcal{H} \rightarrow L^{2}([0, L] \times[0, T])
$$

boundedly iff and only if the rays in $c_{0}$ do not form caustics. This result will be stated more precisely as a theorem in section 4 .

\section{Consequence}

Besides the obvious physical interpretation of the result, the theorem has the following consequence for the inverse problem. One needs to have knowledge of the spectral properties of $\mathcal{F}$ for an efficient inversion algorithm. The theorem shows that $\mathcal{F}$ may have a large spectral radius when caustics are present in the medium. This indicates a source of trouble for inversion algorithms only using local information, for example Gradient based algorithms used to find the minimum of an objective function.

Finally for reference sake we give a list of the Green's functions in various dimensions in a homogeneous medium $c_{0}=$ constant.

$$
\begin{array}{r}
n=1: G_{0}\left(\mathbf{x}_{s} ; \mathbf{x}, t\right)=H\left(c_{0} t-\left|\mathbf{x}-\mathbf{x}_{s}\right|\right) \\
n=2: G_{0}\left(\mathbf{x}_{s} ; \mathbf{x}, t\right)=\frac{c_{0}}{2 \pi} \frac{H\left(c_{0} t-\left|\mathbf{x}-\mathbf{x}_{s}\right|\right)}{\sqrt{c_{0}^{2} t^{2}-\left|\mathbf{x}-\mathbf{x}_{s}\right|^{2}}} \\
n=3: G_{0}\left(\mathbf{x}_{s} ; \mathbf{x}, t\right)=\frac{\delta\left(c_{0} t-\left|\mathbf{x}-\mathbf{x}_{s}\right|\right)}{4 \pi\left|\mathbf{x}-\mathbf{x}_{s}\right|}
\end{array}
$$

\section{The progressing wave expansion}

As promised in the previous section, given that we know $c_{0}$, we can give an asymptotic expansion of the Green's function $G_{0}$. This expansion is known as the progressing wave 
expansion (see [3], page 188). The description naturally leads to the notion of a caustic which we define in this section too.

According to this theory we have an expansion summarized by

$$
G_{0}\left(\mathbf{x}_{s} ; \mathbf{x}, t\right)=a\left(\mathbf{x}_{s} ; \mathbf{x}\right) S\left(t-\tau\left(\mathbf{x}_{a} ; \mathbf{x}\right)\right)+\text { Smoother terms. }
$$

Here the amplitude $a$ satisfies the transport equation

$$
\nabla \cdot\left(a^{2}\left(\mathbf{x}_{s} ; \mathbf{x}\right) \nabla \tau\left(\mathbf{x}_{a} ; \mathbf{x}\right)\right)=0
$$

and the traveltime $\tau$ satisfies the Eikonal equation

$$
\left|\nabla \tau\left(\mathbf{x}_{s} ; \mathbf{x}\right)\right|^{2}=\frac{1}{c(\mathbf{x})^{2}}
$$

Also we included $\mathbf{x}_{s}$ just as a parameter in the above expressions.

Now we will discuss the meaning of the above symbols. $S(\mathbf{x}, t)$ is the most singular term appearing in the Green's function for a homogeneous $(c(x)=$ constant) medium. For example in one dimension $S$ is given by (see the list of Green's functions given in the last section)

$$
S\left(\mathbf{x}_{s}, \mathbf{x}, t\right)=H\left(c_{0} t-\left|\mathbf{x}-\mathbf{x}_{s}\right|\right)
$$

etc. The level curves of the traveltime function $\tau\left(x_{s} ; \mathbf{x}\right)$ are just the wavefronts emanating from the source location $x_{s}$. Alternatively, instead of dealing with wavefronts, we can consider the equivalent concept of rays which are the orthogonal trajectories of the expanding/contracting wavefronts. Then $\tau\left(x_{s} ; \mathbf{x}\right) \equiv \tau(\mathbf{x})$ represents the amount of time that a ray takes to travel from the source $x_{s}$ to $x$. Similarly along the rays there is an associated amplitude $a\left(\mathbf{x}_{s} ; \mathbf{x}\right)$ of the signal. This represents that amplitude of the signal at $\mathbf{x}$ which departed from $x_{s}$ along a ray which intersects $\mathbf{x}$.

Of course implicit in all of the above description of $\tau\left(\mathbf{x}_{s}, \mathbf{x}\right)$ and $a\left(\mathbf{x}_{s} ; \mathbf{x}\right)$ is the fact that we assume that there is only one ray leaving $x_{s}$ and arriving at $x$; i.e. we assume these functions are single valued. This is usually only locally true for even mildly inhomogeneous media. When this fails to be the case there is generally a locus or curve to which there is a ray tangent at every point. For example see figure (5.9) for a distinctive cusp shaped curve with this property. Such a curve is called a caustic. A more precise mathematical description of a caustic is given by reference to the material in [4], section 3.6. For the reader who wants a more precise description of a caustic, there follows a summary.

The Eikonal equation is first order nonlinear partial differential equation (p.d.e.) and we recall how local solutions to such equations may be built geometrically via the method of characteristics as follows.

Consider solving the following first order nonlinear (p.d.e.) described by

$$
f\left(\mathbf{x}, \frac{\partial \tau}{\partial \mathbf{x}}\right)=0, \mathbf{x} \in X
$$




$$
\begin{gathered}
\left.\tau\right|_{\mathcal{M}}\left(\mathbf{x}^{\prime}\right)=g\left(\mathbf{x}^{\prime}\right) \\
\left.\nabla \tau\right|_{\mathcal{M}}\left(\mathbf{x}^{\prime}\right)=h\left(\mathbf{x}^{\prime}\right)
\end{gathered}
$$

for $\tau(\mathbf{x})$, where $\mathbf{x}^{\prime}=\left(x_{1}^{\prime}, \ldots, x_{n-1}^{\prime}\right)$ are appropriate coordinates on the hypersurface $\mathcal{M}$. Let $\nu\left(x^{\prime}\right)$ denote the normal vector field on the hypersurface $\mathcal{M}$. If for all points $x^{\prime}$ on the hypersurface, we have that $f\left(x^{\prime}, \nu\left(x^{\prime}\right)\right) \neq 0$, then $\mathcal{M}$ is called a non characteristic hypersurface for the p.d.e. (2.3).

The method of characteristics says that if $\mathcal{M}$ is a non characteristic hypersurface for the p.d.e (2.3), then a local solution of (2.3) near $\mathcal{M}$ can be constructed as follows.

Consider a neighbourhood (in $R^{n}$ ) of $\mathcal{M}$ which we will call $X$. We will define a solution of (2.3) in an open subset of $X$.

We denote by $T^{*} X$ the cotangent bundle of $X$ which will have coordinates denoted by $(\mathbf{x}, \xi)=\left(x_{1}, \ldots, x_{n} ; \xi_{1}, \ldots \xi_{n}\right)$. Then if we write $\xi=\nabla_{\mathbf{x}} \tau$, we can view $f$ in $(2.3)$ as a function on $T^{*} X$ which we will assume is smooth. Thus trying to find a local solution to equation (2.3) is equivalent to finding the zero set of the so called Hamiltonian function $f(x, \xi)$ through the hypersurface $\mathcal{M}$. In the case of (2.2) the function $f(x, \xi)=|\xi|^{2}-1 / c^{2}(x)$. The solution to this problem is simple to describe, and the interested reader can look in [4] for a justification of the following prescription for a solution.

Associated to the Hamiltonian function $f(\mathbf{x}, \xi)$ is a vector field $H_{f}$ on $T^{*} X$ given in the above coordinates by

$$
H_{f}(\mathbf{x}, \xi)=\sum_{i=1}^{n} \frac{\partial f}{\partial \xi_{i}}(\mathbf{x}, \xi) \frac{\partial}{\partial x_{i}}-\frac{\partial f}{\partial x_{i}}(\mathbf{x}, \xi) \frac{\partial}{\partial \xi_{i}} .
$$

Here $\left\{\left.\frac{\partial}{\partial x_{i}}\right|_{(x, \xi)},\left.\frac{\partial}{\partial \xi_{i}}\right|_{(x, \xi)}\right\}_{i=1}^{n}$ form a basis for the tangent space to $T^{*} X$ at $(\mathbf{x}, \xi)$. Note that $H_{f}(\mathbf{x}, \xi) \cdot f=0$ verifying that $f$ is constant (equal to zero) along the flow of the vector field $H_{f}$.

We consider the curve generated through a point $\mathbf{x}^{\prime}$ on $\mathcal{M}$ by the flow of $H_{f}$. Such a curve is called a bicharacteristic curve. Then consider the union of all the bicharacteristic curves through $\mathcal{M}$ generated by this procedure. This union of curves is a smooth $\mathbf{n}$ dimensional submanifold of $T^{*} X$ which we call $\Lambda$. It turns out that if we only let the bicharacteristic curves flow as long as the resulting $\Lambda$ remains transversal to the fibres in $T^{*} X$, then we will have the following situation. $\left.f\right|_{\Lambda} \equiv 0$ and furthermore $\Lambda$ has local coordinates $(\mathbf{x}, \xi(\mathbf{x}))$, where $\xi(\mathbf{x})$ is a smooth function given by $\xi(\mathbf{x})=\nabla_{\mathbf{x}} \phi$ for some smooth function $\phi(\mathbf{x})$ satisfying $\left.(\phi, \nabla \phi)\right|_{\mathcal{M}}\left(\mathbf{x}^{\prime}\right)=\left(g\left(\mathbf{x}^{\prime}\right), h\left(\mathbf{x}^{\prime}\right)\right)$. Thus our local (unique) solution for $r(\mathbf{x})$ is in fact given by $\phi(\mathbf{x})$. Note that all of the above construction is equivalent (see (4.1)) to solving a system of $2 \mathrm{n}$ ordinary differential equations (generating the Hamiltonian flow) but this lacks the geometrical insight we need to now define a caustic. 
What is interesting to us is what happens when this transversality condition mentioned in the last paragraph fails to hold. This says that the canonical projection $\Pi:(\mathbf{x}, \xi) \rightarrow \mathbf{x}$ from $\Lambda$ into $X$ fails to be a diffeomorphism. i.e. when projecting from a neighbourhood of such a location, then many bicharacteristic curves get "concentrated" in $X$ upon projection. The projection of a bicharacteristic curve into $X$ is known as a characteristic curve or simply a ray. The rays mentioned before are exactly this concept applied to the Eikonal equation. If there is a subset $C$ in $X$ which is the image of the canonical projection from $\Lambda$ and the projection is not a diffeomorphism here, then this curve $\lambda$ is an envelope of rays by definition. Under these circumstances $\lambda$ is known as a caustic. In the case $n=2$ and $\lambda$ being a curve (not necessarily smooth) this curve is known as a caustic curve.

We give the following schematic (since we cannot give a 4 dimensional picture) figure as an example of the above ideas.

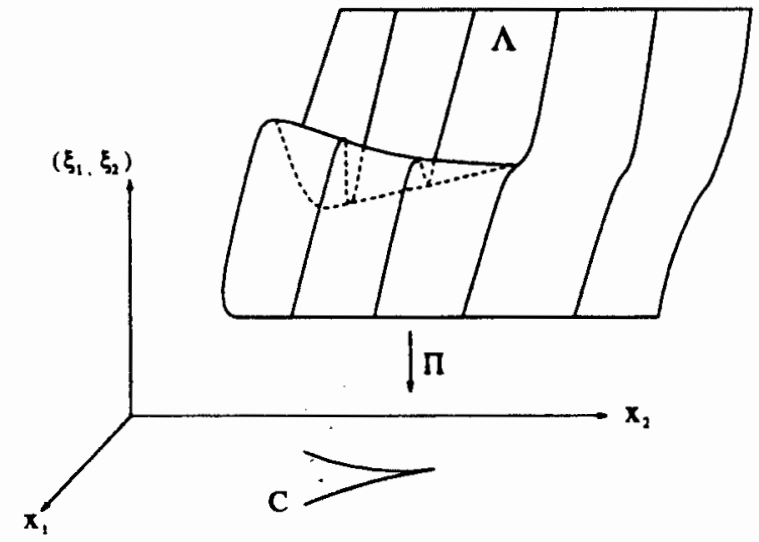

Figure 2.1: The projection $\Pi$ from $\Lambda$ to the caustic $\curlywedge$

\section{A motivational experiment}

We will now give an argument for the main result of the paper. The motivation consists of two parts. First we give a methematical reason which strongly sugests that the result may be true, and then we give a heuristic/physical motivation.

\subsection{The mathematical plausability}

We shall soon see that the forward operator $\mathcal{F}$ is a Fourier Integral Operator [4] and we shall quote a result about such operators which is the basis of the argument here. We state the result here for convenience.

Theorem 3.1 (See - [4], page 154) Let $C \subset T^{*} X \times T^{*} Y-\{0\}$ be a homogeneous canonical relation such that 
1.

$$
\left\{\left(\begin{array}{l}
0 \\
\xi
\end{array}\right),\left(\begin{array}{l}
0 \\
0
\end{array}\right)\right\} \notin T_{(x, \xi, y, \eta)}(C),
$$

2.

$$
\left\{\left(\begin{array}{l}
0 \\
0
\end{array}\right),\left(\begin{array}{l}
0 \\
\eta
\end{array}\right)\right\} \notin T_{(x, \xi, y, \eta)}(C) \text { and }
$$

3. The differential of the projection $C \rightarrow T^{*} X$, (resp. $C \rightarrow T^{*} Y$ ) has rank $\geq \operatorname{dim} X+k$ (resp. $\geq \operatorname{dim} Y+k$ ). Here $k \in \mathbf{Z}$.

Then a Fourier Integral Operator $A \in I_{p}^{m}(X, Y ; C)$ is continous:

$$
H_{C o m p}^{s}(Y) \rightarrow H_{L o c}^{s-m-\frac{1}{4}(\operatorname{dim} X+\operatorname{dim} Y-2 k)}, \forall s, m \in \mathbf{R} .
$$

In our case it will turn out that the forward operator $\mathcal{F} \in I_{1}^{\frac{1}{2}}([0, L] \times[0, T], X, ; \Lambda)$ for $X$ equal to a half space representing the earth's subsurface and $\Lambda$ is generated by a family of bicharacteristics associated to the wave equation - see [12]. It so happens that when caustics develop in the ray field, that the number $k$ mentioned above drops from $k=\operatorname{dim} X=\operatorname{dim} Y$ to some smaller integer. When this happens, the index on the righhand side Sobolev space in (3.1) is strictly less than $s-m$. In our case this means that if a caustic is present then the theorem is telling us that we cannot be sure that $\mathcal{F}$ is continuous from $H^{\frac{1}{2}}(X)$ to $L^{2}([0, L] \times[0, T])$. We can therefore reasonably hope to prove that $\mathcal{F}$ is not continuous in the presence of caustics and consequently that the estimate in the theorem is sharp.

\subsection{The physical plausability}

This argument is based on the following experiment which was first suggested in [2]. It was also this work which first suggested that a theorem like the one mentioned in the last section may be true. In the former thesis [2], formal arguments are presented in support of such a theorem. The ideas presented in this section are also needed for the next section.

Here we consider performing a sequence of separate experiments labelled by an index $j$, whereby a reflectivity $\left(\delta c_{j}\right)$ is introduced to reflect the incident bundle of rays back to the surface of the earth in the form of a plane wave (see figure (3.1)). Before proceeding any further we define more precisely how the "reflectivity" sequence $\delta c_{j}$ is to be chosen. We will restrict our attention to the reflectivity sequence of the form

$$
\delta c_{j}(x)=\chi_{j}(x) e^{i k_{j} \cdot x}
$$


where $\chi_{j}$ is a test function.

Next we have to describe the functions $\chi_{j}$ while we reserve the choice of sequence of vectors $k_{j}$ until later. In order to describe the supports of the reflectivities $\chi_{j}$, let us denote by $\lambda$ the curve prescribing a caustic that develops as a result of tracing rays through the medium from our point source $x_{s}$ - see figure (3.1). Also let us denote by $T$ the interior of the closed ray tube generated by the rays leaving the the source point $x$, and traveling until the caustic curve $\lambda$ is touched by them. We will refer to $\mathcal{T}$ as the "precaustic region". In the next section, we will give a more mathematical description of the precaustic region. For the general element in our sequence we set $\chi_{j}$ equal to the bump function supported on a disk of radius $\nu_{j}$ and with centre $\left(x_{j}, y_{j}\right)$ i.e.

$$
\chi_{j}(x)=\left\{\begin{array}{l}
e^{-\nu_{j} / d_{j}}, \text { if } \sqrt{\left(x-x_{j}\right)^{2}+\left(y-y_{j}\right)^{2}}<\nu_{j} \\
0, \text { if } \sqrt{\left(x-x_{j}\right)^{2}+\left(y-y_{j}\right)^{2}} \geq \nu_{j}
\end{array}\right.
$$

where

$$
d_{j}=\nu_{j}-\sqrt{\left(x-x_{j}\right)^{2}+\left(y-y_{j}\right)^{2}}
$$

The quantities $\nu_{j}$ are chosen to be a strictly monotonic decreasing positive sequence, while $x_{j}, y_{j}$ are chosen such that the distance between the centre point $\left(x_{j}, y_{j}\right)$ and the caustic $\lambda$ is also strictly decreasing. We will require that these disks do not touch the point $x_{a}$ and are also contained in $T$. Furthermore we will refer to the interior of Supp $\chi_{j}$ as $\Omega_{j}$. This situation is depicted in the figure (3.1). Note that the same bundle of rays is intersected ("trapped") by the reflectivity $\delta c_{j}$ in each experiment (i.e. for all $j$ ). Moreover the radius of the disk $\Omega_{j} \longrightarrow 0$ as its centre approaches the caustic (i.e. for all $j$ ).

The fact that it is always possible to "trap" the same bundle of rays and reflect them into a plane wave says something about the shape of the reflector in order that Snell's law be satisfied. That this experimental set up is possible (locally) is simple a consequence of the Implicit Function Theorem (see the appendix for a proof).

The experiment also suggests that the reflectivity sequence reflects arbitrarily large amounts of energy relative to the "size" of the reflector support. Recall that the wave energy in a region is proportional to the $L^{2}$ norm over said region of the amplitude of the signal. In the precaustic region close to a caustic the raytube $\mathcal{T}$ collapses (its diameter tends to zero) and Geometrical Optics (assumed valid here) predicts that the amplitudes "blow up". The reader can verify this immediately by integrating equation (2.1) over the raytube, applying the divergence theorem and noting that $\nabla \tau$ is parallel to the rays generating the raytube. This predicts an arbitrarily large energy density as we approach the caustic and the raytube collapses. However there is a limitation to the above argument as one might have suspected. The above argument relied on geometric optics (ray theory) which is only valid, provided we are on the order of one wave length of the incident field away from the caustic. There exists a more complicated uniform expansion (see [16],[5]) in the neighbourhood of a caustic. 
Our source function $\delta(t)$ in equation (1.6) contains all frequencies, and hence arbjtrarily small wavelengths. Thus we may expect geometrical optics to be valid for these components arbitrarily close to the caustic $\lambda$.

Based on this experiment one may then suspect that the forward map $\mathcal{F}$ is an unbounded map, i.e. that we can find a reflectivity sequence such that the ratio

$$
\frac{\left\|F \delta c_{j}\right\|_{\alpha}}{\left\|\delta c_{j}\right\|_{\beta}} \rightarrow \infty
$$

as the experiment index $j \rightarrow \infty$. The subscripts $\alpha$ and $\beta$ indicate two different norms; the choice of which will become clear in the next section. Our task is to show by example, unboundedness of the latter ratio and hence unboundedness of the forward map. This is the main result of the paper.

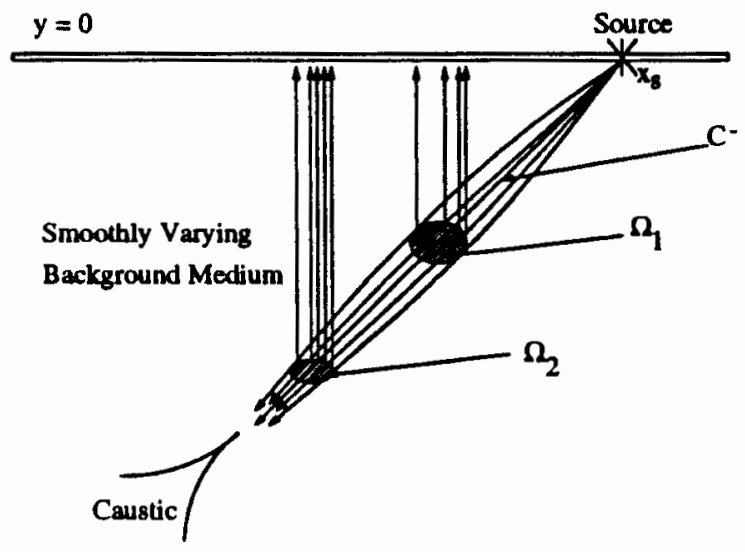

Figure 3.1: A Sequence of reflectivities

\section{Unboundedness of the forward map}

In this section we give a rigoroous proof of the main result of the paper. Let us first establish the hypotheses and notation for this section. Let $\Omega$ be some open subset of $R^{n}$. Then define $H_{c}^{s}(\Omega)$ as the closure test functions $C_{0}^{\infty}(\Omega)$ in $H^{s}$.

Throughout this section, a standing hypothesis will be made. It will be referred to as "the simple ray geometry hypothesis" (SRG), and we will now describe it.

Recall from section 2, that associated to solving the Eikonal equation (2.2) in $R^{n}$, there is a $2 n$ system of ordinary differential equations (O.D.E.'s). This system is known as Hamilton's Equations and solving it amounts to computing a Hamiltonian flow associated 
to the function on $f(\mathbf{x}, \xi)=|\xi|^{2}-c^{-2}(\mathbf{x})$. The system of autonomous O.D.E.'s follows

$$
\begin{array}{r}
\dot{x}=\nabla_{\xi} f(\mathbf{x}(t), \xi(t)) \\
\dot{\xi}=-\nabla_{\mathbf{x}} f(\mathbf{x}(t), \xi(t)) \\
\text { where } \cdot \equiv \frac{d}{d t} .
\end{array}
$$

Let $\theta \in S^{1}$ and denote by $\left(X_{\theta}(t), \xi_{\theta}(t)\right)$ the solution of equations (4.1), which have initial conditions $\left(x_{s}, \frac{\theta}{c\left(x_{0}\right)}\right)$. Note that $t$ is just the ordinary differential equation parameter.

Define the following sets

$$
\begin{aligned}
& \Omega\left(x_{s}\right) \equiv\left\{x: x=X_{\theta}(t), \text { for some } \theta \in S^{n-1}, \text { and } t \in[0, T]\right\} \\
& \Omega^{0}\left(x_{s}, t\right) \equiv\left\{x \in \Omega\left(x_{s}\right): \text { if } x=X_{\theta}(t), \text { then for } 0 \leq t \leq T, X_{\theta}(t)\right. \\
&\text { lies only on the ray } \left.X_{\theta} \text { and } \tau\left(x_{s}, x\right) \leq t\right\} .
\end{aligned}
$$

The boundary points of $\Omega^{0}\left(\mathbf{x}_{s}, t\right)$ belong to caustics, and $\Omega^{0}\left(\mathbf{x}_{s}, T\right)$ defines more precisely what we have been calling the precaustic region.

Now we state the SRG hypothesis:

1. All reflectivity fields are contained in $\Omega^{0}\left(\mathbf{x}_{s}, T\right)$.

2. If $\theta$ is the angle between an incident ray and a reflected ray then $\cos (\theta)>-1$.

3. There are no rays returning to the surface $y=0$ and grazing this surface.

Note that the latter two conditions in the SRG hypothesis ensure according to Rakesh [12] that the forward map $\mathcal{F}$ is a Fourier Integral Operator.

In particular, all of the disks $\Omega_{j}$ defined in section 3 are assumed to lie in the set $\Omega^{0}\left(\mathbf{x}_{s}, T\right)$.

Our next goal will be to establish the following result.

Theorem 4.1 Assume that when rays are traced through a medium, that a caustic $\lambda$ forms. Then under the simple ray geometry hypothesis, the forward map, $\mathcal{F}$ is bounded from $H_{c}^{1 / 2}\left(\Omega^{0}\left(\mathbf{x}_{s}, T\right)\right)$ to $L^{2}([0, L] \times[0, T])$ iff $\partial \Omega^{0}\left(\mathbf{x}_{s}, T\right) \cap \curlywedge=\emptyset$.

The operator $\mathcal{F}$ is in fact a Fourier Integral Operator (FIO) and hence the proof of the theorem requires a knowledge of FIO's. An introduction to this theory can be found in the following references $[4],[17],[8],[6]$. 
We note also that even when caustics are produced, the operator $\mathcal{F}$ (which is now unbounded) still retains the property of being an FIO (of order 1/2) provided that there are no rays leaving the source and returning to graze the plane $y=0$. This latter result is shown in [12]. In the latter case, it also has to be assumed for technical reasons, that the source location $x_{s}$ is strictly below the surface i.e. has positive $y_{s}$ coordinate.

A large part of this section will be devoted to the proof of the theorem. We will need to give some background to establish notation. We will also need several lemmas before we commence the main proof of this theorem.

First, we recall an important fact regarding a subclass of FIO's called Pseudodifferential Operators ( $\Psi$ DO's) - see [4], page 154. Suppose $P$ is a $\Psi D O$ of order $\mu$ then it has the following continuous mapping property on the Sobolev spaces

$$
P: H_{c}^{s} \rightarrow H_{L o c}^{o-\mu} .
$$

It will be useful to study the so called $L^{2}$ normal operator $\mathcal{N}$ which is simply defined by

$$
\mathcal{N} \equiv{ }^{t} \mathcal{F} \circ \mathcal{F}
$$

where ${ }^{t} \mathcal{F}$ is the $L^{2}$ adjoint of $\mathcal{F}$.

Denote by $\mathcal{F}_{j}, \mathcal{N}_{j}$ the restriction of the forward and normal maps respectively to the disks $\Omega_{j}$ defined in section 3 , so that we have

$$
\begin{array}{r}
\mathcal{F}_{j}: H_{c}^{1 / 2}\left(\Omega_{j}\right) \mapsto L^{2}([0, L] \times[0, T]) \\
{ }^{t} \mathcal{F}_{j}: L^{2}([0, L] \times[0, T]) \mapsto H_{c}^{-1 / 2}\left(\Omega_{j}\right) \\
\mathcal{N}_{j}: H_{c}^{1 / 2}\left(\Omega_{j}\right) \mapsto H_{c}^{-1 / 2}\left(\Omega_{j}\right) .
\end{array}
$$

We remark that we will only use $\delta c_{j} \in C_{0}^{\infty}$ but note that this function space is dense in the space of distributions and in particular dense in $H_{c}^{1 / 2}\left(\Omega_{j}\right)$. The idea being that if the forward map were to be discontinuous (unbounded), then it should be unbounded on the test functions too (by the density). In fact we will construct a sequence of test function reflectivities, and then show that the forward map is unbounded on this sequence in the sense stated in the theorem.

We can now start to state and prove the lemmas needed to prove the above theorem.

Lemma (7.1) below gives an asymptotic expansion for $\mathcal{N}_{j}$, which is derived in [14].

For a source location $x_{s}$ and a receiver location $x_{r}$ on $y=0$ define

$$
\phi\left(\mathbf{x}_{s}, \mathbf{x}_{r}, \mathbf{x}\right) \equiv \tau\left(\mathbf{x}_{s}, \mathbf{x}\right)+\tau\left(\mathbf{x}, \mathbf{x}_{r}\right)
$$




$$
\begin{array}{r}
\nabla \phi \equiv|\nabla \phi|(\sin (\psi), \cos (\psi)), \\
\forall \xi \in R^{n}, \hat{\xi} \equiv \frac{\xi}{|\xi|} .
\end{array}
$$

Also given the pair $(\mathbf{x}, \hat{\xi})$, there is a unique location on $y=0, \mathbf{x}_{\tau}=\mathbf{x}_{\tau}(\mathbf{x}, \hat{\xi})$ defined as follows. Consider a ray leaving the source $x_{s}$, arriving at $x$, and reflecting so that $-\hat{\xi}$ is the bisector of the angle $\theta\left(x_{s}, x_{r}, \mathbf{x}\right)$ made by the incident ray and the reflected ray emerging at $x_{r}$ - see figure (4.2).

Denote by $\hat{\delta} c_{j}$ the Fourier transform of $\delta c_{j}$. Then we have

Lemma 4.1

$$
\mathcal{N}_{j} \delta c_{j}(\mathbf{x})=\int_{\mathbf{R}^{2}} d \xi e^{i \mathbf{x} \cdot \boldsymbol{\xi}} n_{j}(\mathbf{x}, \xi) \hat{\delta} \hat{c}_{j}(\xi)
$$

where

$$
\left\{\begin{array}{l}
n_{j}(\mathbf{x}, \xi)=n_{0, j}(\mathbf{x}, \xi)+\mathcal{O}(\mathbf{x}, \hat{\xi}) \\
n_{0, j}(\mathbf{x}, \xi)=\left.|\xi| \frac{\left(c^{-\frac{3}{2}}(\mathbf{x}) m\left(\mathbf{x}_{,}, \mathbf{x}_{r}, t_{r}\right) a\left(\mathbf{x}_{s}, \mathbf{x}\right) a\left(\mathbf{x}_{r}, \mathbf{x}\right)\right)^{2}}{\left(1+\cos \theta\left(\mathbf{x}_{\imath}, \mathbf{x}_{r}, \mathbf{x}\right)\right)^{\frac{3}{2}} \frac{\partial_{\phi}}{\partial x_{r}}\left(\mathbf{x}_{\boldsymbol{r}}, \mathbf{x}_{r}, \mathbf{x}\right)}\right|_{\mathbf{x}_{r}=\mathbf{x}_{r}(\mathbf{x}, \hat{\xi})} .
\end{array}\right.
$$

Lemma (4.1) says that the normal operator $\mathcal{N}_{j}$ is a Pseudodifferential operator ( $\Psi$ DO) [15] (of order 1).

The following result will become important to us soon.

Lemma 4.2 There exists a conic set $\Xi$ in $R^{2}-\{0\}$ based at 0 , and a sequence of disks $\Omega_{j}$ such that

$$
\inf _{\mathbf{x} \in \Omega_{j}} \frac{n_{0, j}(\mathbf{x}, \xi)}{|\xi|} \rightarrow \infty \text { as } j \rightarrow \infty, \forall \hat{\xi} \in \Xi \cap S^{1} .
$$

Proof: Define $\vartheta_{j}(\mathbf{x})$ as the characteristic function of the disk $\Omega_{j}$. Then according to Lemma (4.1), we have

$$
\frac{n_{j, 0}(\mathbf{x}, \xi)}{|\xi|}=\left.\frac{\left(c^{-\frac{3}{2}}(\mathbf{x}) m\left(\mathbf{x}_{s}, \mathbf{x}_{r}, t_{r}\right) a\left(\mathbf{x}_{s}, \mathbf{x}\right) a\left(\mathbf{x}_{r}, \mathbf{x}\right)\right)^{2}}{\left(1+\cos \theta\left(\mathbf{x}_{s}, \mathbf{x}_{r}, \mathbf{x}\right)\right)^{\frac{3}{2}} \frac{\partial \psi}{\partial \mathbf{x}_{r}}\left(\mathbf{x}_{s}, \mathbf{x}_{r}, \mathbf{x}\right)} \vartheta_{j}(\mathbf{x})\right|_{\mathbf{x}_{r}=\mathbf{x}_{r}(\mathbf{x}, \hat{\xi})} .
$$


The function $\vartheta_{j}$ has been appended to emphasise the fact that it is understood, $\mathrm{x} \in \boldsymbol{\Omega}_{j}$, where $\Omega_{j}$ is defined as in section 3 . Towards the end of this proof we will modify the definition of $\boldsymbol{\Omega}_{j}$ slightly.

The amplitude term $a\left(x_{s}, x\right)$ in (4.6) is unbounded for an incoming wave with unbounded frequency content as discussed at the end of section 3 . The same is true for $a\left(x_{r}, \mathbf{x}\right)$ because it satisfies the transport equation (2.1) which can be written as an O.D.E.

$$
\frac{d}{d t} a\left(\mathbf{x}_{r}, \mathbf{x}(t)\right)+\frac{1}{2} \nabla \cdot \nabla \tau\left(\mathbf{x}_{r}, \mathbf{x}(t)\right)=0 .
$$

Since $a\left(x_{\tau}, \mathbf{x}\right)$ obeys an ordinary differential equation (transport equation) with a nonzero initial condition we can bound this amplitude from below by some constant.

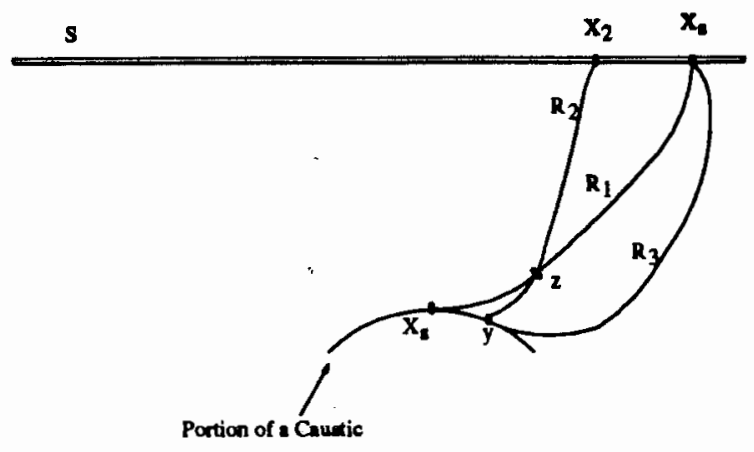

Figure 4.1: Argument for boundedness of $\frac{\partial \psi}{\partial x_{r}}$

With regard to the term $\vartheta_{j}(x) \frac{\partial \psi}{\partial x_{r}}\left(\mathbf{x}_{a}, \mathbf{x}_{r}, \mathbf{x}\right)$, we must be sure that this term does not become large with increasing $j$. For then it could begin to compete with the amplitude terms, and the unboundedness the ratio in (4.6) would no longer be obvious. Therefore we give the following argument to prove that if we restrict the cone in which $\xi$ varies then we can always avoid this situation. Pick a point $\mathbf{x}_{c}$ on the caustic $\curlywedge$ such that there is a ray $R_{1}$ starting at $x_{c}$ and intersecting the plane $y=0$ transversally at $x_{0}-$ this is possible by the definition of a caustic. Denote by $\alpha_{1}$ the takeoff angle from the vertical made by $R_{1}$. Now because $\Omega^{\circ}\left(x_{s}, T\right)$ is an open subset of $y>0$, there must exist a point $y \in \curlywedge$ sufficiently close to $x_{c}$, and an angle $\alpha_{2}$ sufficiently close to $\alpha_{1}$, such that the ray $R_{2}$ departing from $\mathrm{y}$ at an angle of $\alpha_{2}$ must intersect $R_{1}$ at some point $z \in \Omega^{0}\left(\mathrm{x}_{3}, T\right)$ and also $R_{2}$ stays within $\Omega^{0}\left(x_{s}, T\right)$ and intersects $y=0$ at some point $x_{2}$ transversally. The latter observation is true because of continuous (smooth) dependence of solutions to O.D.E.'s (rays) on the their initial data. If $x_{2}=x_{s}$ then the SRG hypothesis is violated at $z$. Therefore we can assume that the ray $R_{2}$ emerges on $y=0$ at $\mathbf{x}_{2} \neq \mathbf{x}_{s}$. Now we have the following situation (see figure (4.1) ): there are two rays departing from $y$; namely the ray $R_{2}$ and the ray $R_{3}$ defined by the ray connecting $x_{s}$ to $y$ (this exists since $y$ is a caustic point). Let $R_{3}$ have takeoff angle $\alpha_{3}$, so that the rays $R_{2}$ and $R_{3}$ depart from $y$ with take-off angles $\alpha_{2}, \alpha_{3}$ respectively, and arrive back on $y=0$ at two different locations. Therefore there must exist a ray departing from $y$ with take-off angle $\alpha^{*} \in\left(\alpha_{2}, \alpha_{3}\right)$ and intersecting $y=0$ at some point $x_{\tau}^{*}$ such that $\frac{\partial x_{r}}{\partial \psi}\left(x_{s}, x_{r}^{*}, y\right) \neq 0$. In fact $\mathbf{y}$ could be any 
caustic point, so $\frac{\partial \psi}{\partial \mathbf{x}_{r}}\left(\mathbf{x}_{s}, \mathbf{x}_{r}^{*}, \mathbf{x}_{c}\right)$ is finite, provided the $\xi$ varies in a sufficiently small conic set $\Xi$ which has axis equal to the line that makes an angle $\alpha^{*}$ with the vertical. Therefore $\frac{\partial \psi}{\partial x_{r}}\left(\mathbf{x}_{s}, \mathbf{x}_{r}(\mathbf{x}, \hat{\xi}), \mathbf{x}\right)$ is bounded independent of $j$ if we restrict the cone in which $\xi$ varies, or correspondingly restrict the interval in which $x_{r}$ varies.

Also, for any $j$, we can always pick a suitable $L$ and $T$ such that $m\left(\mathbf{x}_{r}(\mathbf{x}, \hat{\xi}), t_{r}\right)=1$, whenever $x \in \Omega_{j}, \xi \in \Xi \cap S^{1}$ and $t_{r} \in[0, T]$.

Clearly all other terms in (4.6) are such that we can find a sequence of positive numbers $\left\{C_{j}\right\}$, with $C_{j} \longrightarrow \infty$, so that for $(\mathbf{x}, \xi) \in \Omega_{j} \times \Xi$, then $n_{0, j}(\mathbf{x}, \xi) /|\xi|>C_{j}$. This completes the proof of the lemma.

Now define a new operator $\hat{\mathcal{N}}_{j}$, which is "extension" of $\mathcal{N}_{j}$ got by giving it the same symbol as $\mathcal{N}_{j}$ in $\Omega_{j} \times \Xi$ and extending it to all of $\Omega_{j} \times S^{1}$ so that its principle symbol $\hat{n}_{0, j}(\mathbf{x}, \hat{\xi})$ satisfies the inequality

$$
\inf _{\mathbf{x} \in \Omega_{j}, \hat{\xi} \in S^{1}} \hat{n}_{0, j}(\mathbf{x}, \hat{\xi}) \geq C_{j} / 2
$$

and such that $\hat{n}_{j}$ is still a symbol of order one. This construction is trivial to carry out, and makes $\hat{\mathcal{N}}_{j}$ a strictly elliptic $\Psi \mathrm{DO}$ of order zero. Thus if $\delta c_{j}$ has Fourier transform rapidly decaying outside of the cone $\Xi$, then $\mathcal{N}_{j} \delta c_{j}(\mathbf{x})=\hat{\mathcal{N}}_{j} \delta c_{j}(\mathbf{x})$ modulo a smooth function of $\mathbf{x}$.

From now on in our demonstration of unboundedness of the forward map we will assume that our reflectivities have rapidly decaying Fourier transform outside of $\Xi$ and we will accordingly replace $\mathcal{N}_{j}$ by $\hat{\mathcal{N}}_{j}$. Under this assumption $\hat{\mathcal{N}}_{j}$ differs from $\mathcal{N}_{j}$ by a smoothing operator. However we continue to write $\mathcal{N}_{j}$ in place of $\mathcal{N}_{j}$ for convenience of notation.

\section{Proof of Theorem (4.1):}

$[\Rightarrow]$ We first prove the only if part of the theorem. To prove it we will take the contrapositive point of view and assume that $\partial \Omega^{0}\left(\mathbf{x}_{s}, T\right) \cap \curlywedge \neq \emptyset$. Then this part of the theorem will be proved by showing that $\mathcal{F}$ is unbounded under this assumption.

In the following $\langle\cdot, \cdot\rangle$ will denote the usual $L^{2}$ inner product and $\langle\cdot, \cdot\rangle_{(r,-r)}$ will denote the usual Sobolev space duality pairing. Also $\|\cdot\|_{s}$ will denote the norm in the Sobolev space $H^{s}$.

The key to the proof of the theorem is Gårding's Inequality [15] (page 55), which says that

$$
\left\langle\mathcal{N}_{j} \delta c_{j}, \delta c_{j}>\geq C_{1, j}\left\|\delta c_{j}\right\|_{\frac{1}{2}}^{2}+\left\langle S_{j} \delta c_{j}, \delta c_{j}>_{(r, r)}\right.\right.
$$

where $S_{j}$ is a smoothing operator that arises in the proof of the Gårding Inequality. This says we have

$$
\left\|\mathcal{F}_{j} \delta c_{j}\right\|_{0}^{2}=\left\langle\delta c_{j}, \mathcal{N}_{j} \delta c_{j}>\geq C_{1, j}\left\|\delta c_{j}\right\|_{\frac{1}{2}}^{2}-C_{r, j}\left\|\delta c_{j}\right\|_{r}^{2}\right.
$$


We will apply Gårding's Inequality to $\mathcal{N}_{j}$ (notice that our error of replacing $\hat{\mathcal{N}}_{j}$ by $\mathcal{N}_{j}$ could be absorbed by the smoothing operator $S_{j}$, under our assumption that our reflectivities have Fourier transform decaying rapidly outside of the cone $\Xi$ ).

The proof of Gårding's Inequality says that $r$ can be any real number, and $C_{1, j}$ is given by

$$
C_{1, j}=\frac{1}{2} \inf _{\mathbf{x} \in \Omega_{j, \hat{\xi} \in S^{1}}} \frac{n_{0, j}(\mathbf{x}, \hat{\xi})}{|\xi|}
$$

(we have just shown that this sequence is unbounded).

Next let us fix $r=0$.

Our goal now is to show that we can construct $\delta c_{j}$ (by choosing $\left|k_{j}\right|$ appropiately) such that the first member of the right hand side of $(4.8)$ is the dominant term.

Let $\epsilon>0$ and for any $j \in N$ we want to find $\delta c_{j}$ such that

$$
r_{j} \equiv \frac{\left\|\delta c_{j}\right\|_{0}}{\left\|\delta c_{j}\right\|_{\frac{1}{2}}} C_{0, j}<\epsilon, \forall j .
$$

Having succeeded in showing this is possible then the first term on the right hand side of (4.8) is dominant and we get unboundedness of the quantity

$$
R_{j} \equiv \frac{\left\langle\delta c_{j}, \mathcal{N}_{j} \delta c_{j}\right\rangle}{\left\|\delta c_{j}\right\|_{\frac{1}{2}}^{2}}=\frac{\left\|\mathcal{F} \delta c_{j}\right\|_{0}^{2}}{\left\|\delta c_{j}\right\|_{\frac{1}{2}}^{2}} .
$$

Now we need just prove that we can find a reflectivity sequence such that the relation (4.10) holds.

Recall that our reflectivity sequence has the form

$$
\delta c_{j}(\mathbf{x})=\chi_{j}(\mathbf{x}) e^{i k_{j} \cdot \mathbf{x}}
$$

where $\chi_{j} \in C_{0}^{\infty}\left(\Omega_{j}\right)$. Then we have that

$$
\begin{array}{r}
\left\|\delta c_{j}\right\|_{\frac{1}{2}}^{2}=\int \lambda^{\frac{1}{2}}(\zeta)\left|\delta \hat{c}_{j}(\zeta)\right|^{2} d \zeta \\
\text { where } \lambda(\zeta)=\left(1+|\zeta|^{2}\right)^{\frac{1}{2}} \\
=\int \lambda^{\frac{1}{2}}(\zeta)\left|\int e^{i \mathbf{x} \cdot\left(k_{j}-\zeta\right)} \chi_{j}(\mathbf{x}) d x\right|^{2} d \zeta \\
=\int \lambda^{\frac{1}{2}}\left(\zeta+k_{j}\right)\left|\int e^{-i x \cdot \zeta} \chi_{j}(\mathbf{x}) d x\right|^{2} d \zeta . \\
\text { So, }\left\|\delta c_{j}\right\|_{\frac{1}{2}}^{2}=\int \lambda^{\frac{1}{2}}\left(\zeta+k_{j}\right)\left|\hat{\chi}_{j}(\zeta)\right|^{2} d \zeta .
\end{array}
$$


Now use Peetre's Inequality [13] (page 21),

$$
\lambda^{s}(\omega) \leq 2^{|\alpha|} \lambda^{|\alpha|}(\omega-\eta) \lambda^{s}(\eta)
$$

with $s=\frac{1}{2}, \omega=k_{j}, \eta=k_{j}+\zeta$ to get

$$
\begin{aligned}
\left\|\delta c_{j}\right\|_{\frac{1}{2}}^{2} \geq 2^{-\frac{1}{2}} \lambda^{\frac{1}{2}}\left(k_{j}\right) & \int \lambda^{-\frac{1}{2}}(\zeta)\left|\hat{\chi}_{j}(\zeta)\right|^{2} d \zeta \\
= & 2^{-\frac{1}{2} \lambda^{\frac{1}{2}}}\left(k_{j}\right)\left\|\chi_{j}\right\|_{-\frac{1}{2}}^{2}
\end{aligned}
$$

Therefore we have

$$
\begin{aligned}
r_{j} & \leq C_{0, j} 2^{\frac{1}{2}} \lambda\left(k_{j}\right)^{-\frac{1}{2}} \frac{\left\|\delta c_{j}\right\|_{0}^{2}}{\left\|\chi_{j}\right\|_{-\frac{1}{2}}^{2}} \\
& =C_{0, j} 2^{\frac{1}{2}} \lambda\left(k_{j}\right)^{-\frac{1}{2}} \frac{\left\|\chi_{j}\right\|_{0}^{2}}{\left\|\chi_{j}\right\|_{-\frac{1}{2}}^{2}} .
\end{aligned}
$$

Next we choose the magnitude of $k_{j}$ sufficiently large so that

$$
2^{\frac{1}{2}} \lambda\left(k_{j}\right)^{-\frac{1}{2}} \frac{\left\|\chi_{j}\right\|_{0}^{2}}{\left\|\chi_{j}\right\|_{-\frac{1}{2}}^{2}} C_{0, j}<\epsilon, \forall j
$$

holds; thus yielding $r_{j}<\epsilon$ independently of $j$. Note this relies on the fact that the ratio $\frac{\left\|x_{j}\right\|_{0}^{2}}{\left\|x_{j}\right\|_{-\frac{1}{2}}^{2}}$ is independent of $k_{j}$. This proves the "only if" part of the theorem.

$[\Leftarrow]$ The "if" part of the theorem is trivial, because if $\partial \Omega^{0}\left(x_{s}, T\right) \cap \wedge=\emptyset$, we know that the normal operator ${ }^{t} \mathcal{F} \mathcal{F}$ is a $\Psi$ DO (see [16], [14]). This means that it is a continuous operator on the Sobolev spaces, as remarked before. The result follows, and the proof of the theorem is complete.

This was the hardest result to prove in this section and now we can improve on Theorem 1 in the form of the following corollories.

Corollary 4.1 Assume that when rays are traced through a medium that a caustic $ᄉ$ forms. Let $\Omega$ be a subset of the halfspace $y>0$. Then under the SRG hypothesis we have that the forward map $\left.\mathcal{F}\right|_{\Omega}$ is bounded from $H_{c}^{1 / 2}(\Omega) \rightarrow L^{2}([0, L] \times[0, T])$ iff $\Omega \cap \curlywedge=\emptyset$.

Proof: In general $\left.\mathcal{F}\right|_{\Omega}$ is a locally finite sum of FIO's $\left.\mathcal{F}\right|_{\Omega}=\sum_{i=1}^{N} \mathcal{F}_{i}$, where each $\mathcal{F}_{i}$ has canonical relation given by a canonical graph (see [16],[11]). The proof now follows from Theorem 3.1 and the proof of Theorem 4.1. 
Corollary 4.2 Assume that when rays are traced through a medium that a caustic $\lambda$ forms. Let $\Omega$ be an open subset of the "post-caustic" region i.e. the multiply covered region of the medium. Then $\left.\mathcal{F}\right|_{\Omega}$ is bounded from $H_{c}^{1 / 2}(\Omega) \rightarrow L^{2}([0, L] \times[0, T])$ iff $\Omega \cap \Lambda=\emptyset$.

Proof: As in the previous corollary $\mathcal{F}_{\Omega}=\sum_{i=1}^{N} \mathcal{F}_{i}$ holds. The proof of sufficiency follows from the previous corllary. For the necessity, we construct a reflectivity sequence $\delta c_{j} \rightarrow$ $c \in H_{c}^{1 / 2}(\Omega)$ as in theorem 4.1. However this time let us arrange for the following to hold. At any point $x \in \Omega$ there several sheets of the Lagrangian $\Lambda$ manifold associated to $\mathcal{F}$ lying over $x$ i.e. $\pi^{-1}(x)=\left\{\lambda_{1}, \cdots, \lambda_{p}\right\}$ where each $\lambda_{j}$ is associated to a different sheet of $\Lambda$. Let us arrange that the wavefront set (see [9] for definition) of $c$ only intersects one sheet of the Lagrangian $\Lambda$ :

$$
W F(c) \bigcap \Lambda \text { is connected. }
$$

This sheet corresponds to the canonical relation of the FIO $\mathcal{F}_{1}$ for example. The proof now follows as in theorem 4.1 because

$$
\mathcal{N}_{j} \delta c_{j}={ }^{t} \mathcal{F}_{1} \mathcal{F}_{1} \delta c_{j}+S_{j} \delta c_{j}
$$

where $S_{j}$ is a smoothing operator which can be absorbed into the smoothing operator appearing in the application of Gårding's inequality in theorem 4.1. The proof is completed by applying theorem 4.1 . to the operator $\mathcal{F}_{1}$.

Remark: Note that the question of boundedness as in the above theorem and corollaries when a reflectivity field has its support only on a caustic is still an open (no pun intended!) question.

Remark: Note that in theorem 4.1 and the above corollaries we have an interesting result when caustics form in a medium. One might be concerned about how likely it is that a caustic will form. In the paper by White [18], it is argued from a stochastic point of view, that even in a mildly heterogeneous medium a caustic will form (after a sufficiently long time) with probability one! 


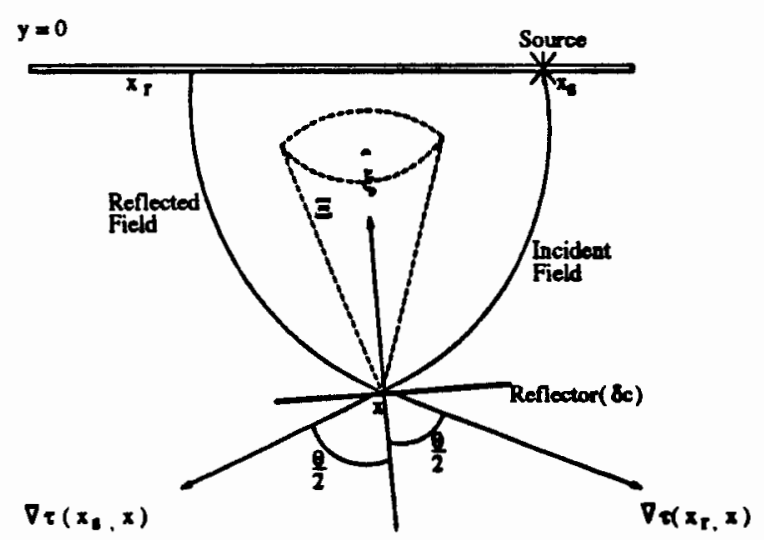

Figure 4.2: Snell's Law and the definition of $x_{r}(x, \hat{\xi})$.

\section{$5 \quad$ Numerical Tests}

There is also another forward map related to $\mathcal{F}$. It is the map resulting from the more general source $f(t) \delta\left(x-x_{s}\right)$ on the right hand side of (1.6). We call this forward map $\mathcal{F}[f]$ to emphasize its dependence on $f$. Since $\mathcal{F}$ results from an impulsive source $\delta(t) \delta\left(x-x_{s}\right)$, then we must have $\mathcal{F}[f]=\mathcal{F} *_{t} f(\cdot)$. It is obvious then that $\mathcal{F}[f]$ is unbounded too for a suitable source function $f \in L^{2}$. The choice of $f$ is important in the numerical experiments, since it is the modified map $\mathcal{F}[f]$ that we will implement in this section. For convenience of notation, we will write $\mathcal{F}$ in place of $\mathcal{F}[f]$ throughout this section.

In order not to have to figure out what reflectivity fields give a large ratio $R_{j}$ (defined by (4.11) with $\mathcal{F}$ replaced by $\mathcal{F}[f]$ ), we decided to to run a power method, so that the computer works this out. First though, we need to clarify how the power method applied in our case.

Define

$$
\mathcal{N}_{j}^{h} \equiv \mathcal{F}_{j}^{*} \circ \mathcal{F}_{j}
$$

where $\mathcal{F}_{j}^{*}$ refers to the $H_{c}^{\frac{1}{2}}\left(\Omega_{j}\right)$ Hilbert space adjoint of $\mathcal{F}_{j}$.

This quantity is of interest because of the following relation

$$
\left\|\mathcal{F}_{j} \delta c_{j}\right\|_{0}^{2}=<\mathcal{N}_{j}^{h} \delta c_{j}, \delta c_{j}>_{\frac{1}{2}}
$$

Therefore we have that the largest value of $\left\|\mathcal{F}_{j} \delta c_{j}\right\|_{0}^{2}$ in the unit sphere of $H_{c}^{\frac{1}{2}}\left(\Omega_{j}\right)$ is equal to the largest Eigenvalue of $\mathcal{N}_{j}^{h}$ (recall that computationally $\mathcal{N}_{j}^{h}$ as a linear operator is some finite dimensional matrix!) 
It is this Hilbert space normal operator that we apply the power method to. So next we calculate $\mathcal{F}_{j}^{*}$ in terms of quantities that we can implement.

Define the following operator on Sobolev spaces

$$
\Lambda \equiv(I-\nabla \cdot \nabla)^{\frac{1}{2}}
$$

where $I$ is the appropiate identity operator, for the Sobolev space in question.

\section{Lemma 5.1}

$$
\mathcal{N}_{j}^{h}=\Lambda^{-1} \circ{ }^{t} \mathcal{F}_{j} \circ \mathcal{F}_{j}
$$

Proof: Let $\delta c_{j} \in H_{c}^{\frac{1}{2}}\left(\Omega_{j}\right), v_{j} \in L^{2}([0, L] \times[0, T])$. Then we have

$$
\left\langle\mathcal{F}_{j} \delta c_{j}, v_{j}\right\rangle_{0}=\left\langle\delta c_{j},{ }^{t} \mathcal{F}_{j} v_{j}\right\rangle_{\left(\frac{1}{2},-\frac{1}{2}\right)}
$$

Note that the right hand side of the last equation is just a bounded linear functional on $H_{c}^{\frac{1}{2}}(\Omega)$ and so by the Riesz representation theorem we are guaranteed the existence of the operator $\mathcal{F}_{j}^{*}$ such that

$$
\left.\left\langle\mathcal{F}_{j} \delta c_{j}, v_{j}\right\rangle_{0}=<\delta c_{j}, \mathcal{F}_{j}^{*} v_{j}\right\rangle_{\frac{1}{2}}
$$

Thus we get

$$
\begin{array}{r}
<\delta c_{j}, \mathcal{F}_{j}^{*} v_{j}>_{\frac{1}{2}}=\left\langle\delta c_{j},{ }^{t} \mathcal{F}_{j} v_{j}\right\rangle_{\left(\frac{1}{2},-\frac{1}{2}\right)} \\
\Rightarrow\left\langle\Lambda^{\frac{1}{2}} \delta c_{j}, \Lambda^{\frac{1}{2}} \mathcal{F}_{j}^{*} v_{j}\right\rangle_{0}=\left\langle\delta c_{j},{ }^{t} \mathcal{F}_{j} v_{j}\right\rangle_{\left(\frac{1}{2},-\frac{1}{2}\right)} \\
\Rightarrow\left\langle\delta c_{j}, \Lambda \mathcal{F}_{j}^{*} v_{j}\right\rangle_{\left(\frac{1}{2},-\frac{1}{2}\right)}=\left\langle\delta c_{j},{ }^{t} \mathcal{F}_{j} v_{j}\right\rangle_{\left(\frac{1}{2},-\frac{1}{2}\right)} \\
\Rightarrow \mathcal{N}_{j}^{h}=\mathcal{F}_{j}^{*} \mathcal{F}_{j}=\Lambda^{-1}{ }^{t} \mathcal{F}_{j} \mathcal{F}_{j} .
\end{array}
$$

This concludes the proof.

Four experiments were performed and in each case the following was the procedure. The (Hilbert space) normal operator was composed of several operators reflecting the result of the last lemma. These maps in turn were implemented by finite difference codes or in the case of the $\Lambda$ operator this was done by using discrete Fourier transforms. This normal map was run many times with the output of the normal map being normalized and then used as the input again. In this way the power method was finding the Eigenvector (reflectivity) corresponding to the largest Eigenvalue of $\mathcal{N}_{j}^{h}$ (corresponding to a large value of $R_{j}$ ). 
It was also noticed that in all cases that as we moved $\Omega_{j}$ away from the source the ratio $\boldsymbol{R}_{\boldsymbol{j}}$ decreased until it started to approach the caustic when it started to increase in the vicinity thereof again. On the other side of the caustic it of course decreased again. It was found that just off centre from the peak of the caustic (see figure 5.9) that $R_{j}$ was larger than at the peak which also seems interesting; perhaps some interference is responsible for this effect. This is the location which we chose for our tests (see figures (5.1),(5.2), etc). Note that we implemented $\mathcal{N}_{j}$, which is $\mathcal{N}$ restricted to $\Omega_{j}$, by injecting $\Omega_{j}$ into a larger grid, applying $\mathcal{N}$ and then projecting the result onto $\Omega_{j}$.

The four experiments performed were the following. In the first experiment a peak frequency of $126 \mathrm{~Hz}$ was used for the source in a medium which has a lens (low velocity zone) in the background velocity $c_{0}$ which forms a caustic (see figure (5.9)). The reflectivity got from the power method is shown in figure (5.1) and the output of $\mathcal{F}$ is shown in figure (5.3). The second experiment is identical except the lens is removed to leave a constant background of $1500 \mathrm{~m} / \mathrm{s}$ (the lens had a minimum velocity of $1200 \mathrm{~m} / \mathrm{s}$ approx.) The results are shown in figures (5.2) and (5.4). The third experiment is the same as the first except the source peak frequency is reduced to $63 \mathrm{~Hz}$; see figures (5.5) and (5.7) for the results. The fourth experiment is the corresponding lower frequency experiment as the second; see figures (5.6) and (5.8) for the results.

The shape of the reflectivity got in these experiments makes sense because it is oscillating in a vertical direction which is the direction of the impinging rays for the most part and hence it will reflect most of the energy vertically back to the receivers. The output of $\mathcal{F}$ is easily interpreted too; we see that it has several "layers" in it corresponding to reflection from the various peaks in the reflectivity.

For the sources we used Ricker wavelets, which roughly speaking is the second derivative of a Gaussian function. More precisely, if $\nu_{p k}$ denotes the peak frequency of the wavelet,

$$
f(t)=\frac{d}{d t^{2}} e^{-\omega t^{2}} ; \omega=\left(\pi \nu_{p k}\right)^{2} .
$$

See figure (5.10) for an example of a ricker wavelet. In our tests the wavelets were normalized so that the sup norm of their Fourier Transform was unity.

The results for the maximum values of $R_{j}$ are indicated in the captions. These results indicate a stronger reflection in the vicinity of the caustic and this is enhanced at a higher frequency as expected since the theory was based upon high frequency asymptotics. The next step to carry out is to refine the grid so that we can have smaller reflectivities which can be finely sampled to oscillate rapidly as seems to be what the reflectivity "wants to do" in order to get a large value of $R_{j}$. The unfortunate aspect of this is that the computational time goes up by a factor of around 8 every time we halve the grid size and time step. Also we need to consider different sources in view of the comment at the end of the last section.

If one examines the asymptotic behaviour of the wave field theoretically in the neighbourhood which actually includes a piece of a cusp shaped caustic, as was done in [10] 
one finds that the amplitude is larger than the field away from the caustic by a factor of $\omega^{1 / 6}$ if our probing wave is monochromatic of frequency $\omega$. This is not a very large exponent and thus indicates that quite a large frequency may be needed to actually see the effect of our theorem. This again is limited by computational resources; especially memory considerations will be important if we are to sufficiently sample our source function.

The following pictures of $\mathcal{F} \delta c$ have time along the vertical axis (increasing downward) while the $x_{r}$ coordinate is the horizontad coordinate. For the reflectivities, the vertical axis is the " $y$ " spatial axis, and the horizontal axis is the " $x$ " spatial axis. 


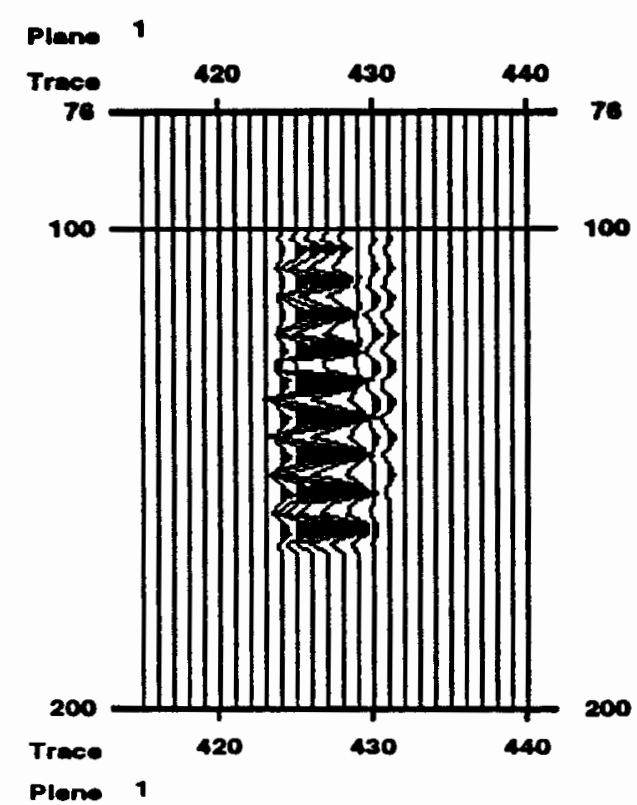

Figure 5.1: Reflectivity $\delta c_{j}$ - Pk Freq. $126 \mathrm{~Hz}$; Lens Medium; $R_{j}=0.10 \mathrm{e}-2$. Horizontal axis $=\mathbf{x}$, vertical axis $=\mathbf{y}$.

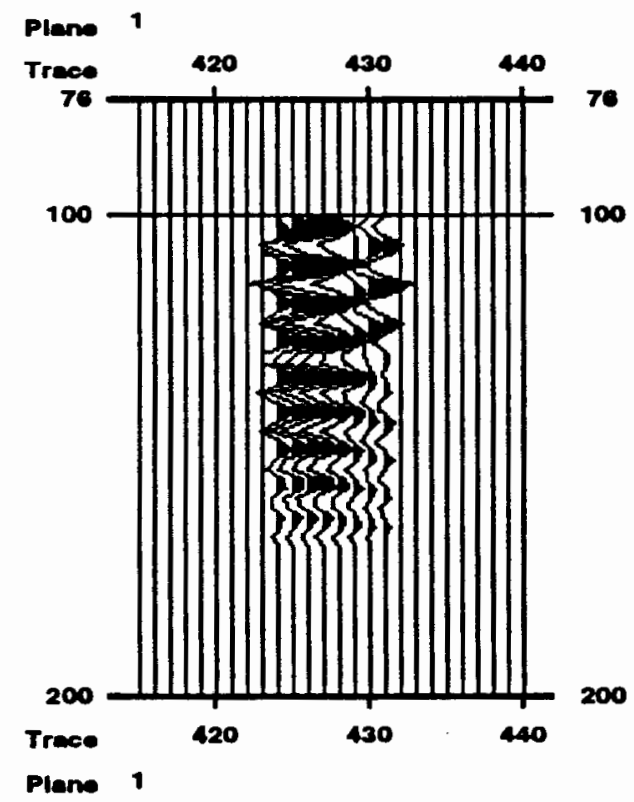

Figure 5.2: Reflectivity $\delta c_{j}$ - Pk Freq. $126 \mathrm{~Hz}$; No Lens; $R_{j}=0.21 \mathrm{e}-3$. Horizontal axis $=$ $\mathrm{x}$, vertical axis $=\mathrm{y}$. 


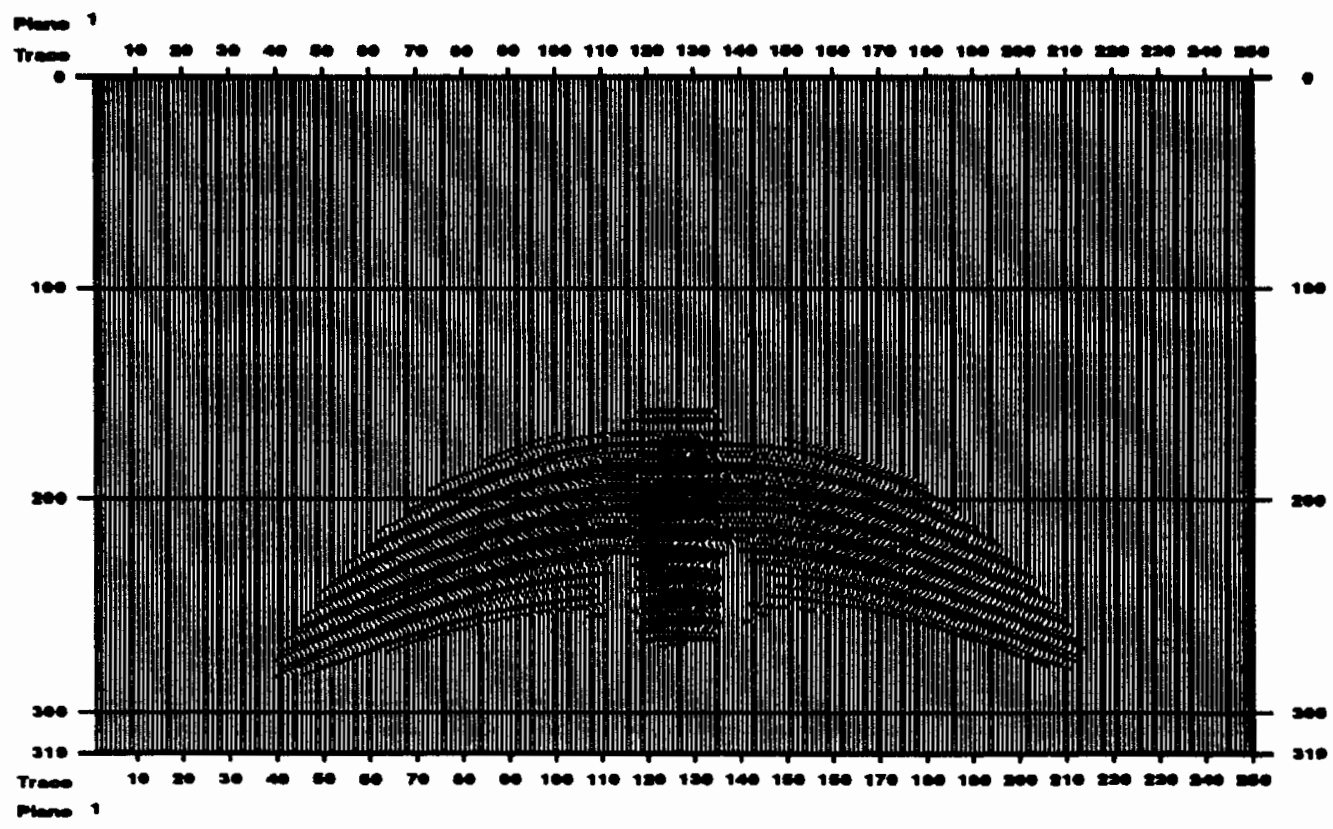

Figure 5.3: $\mathcal{F}_{j} \delta c_{j}-$ Pk Freq. $126 \mathrm{~Hz}$; Lens. Horizontal axis $=x_{r}$, Vertical axis $=$ time $t_{r}$.

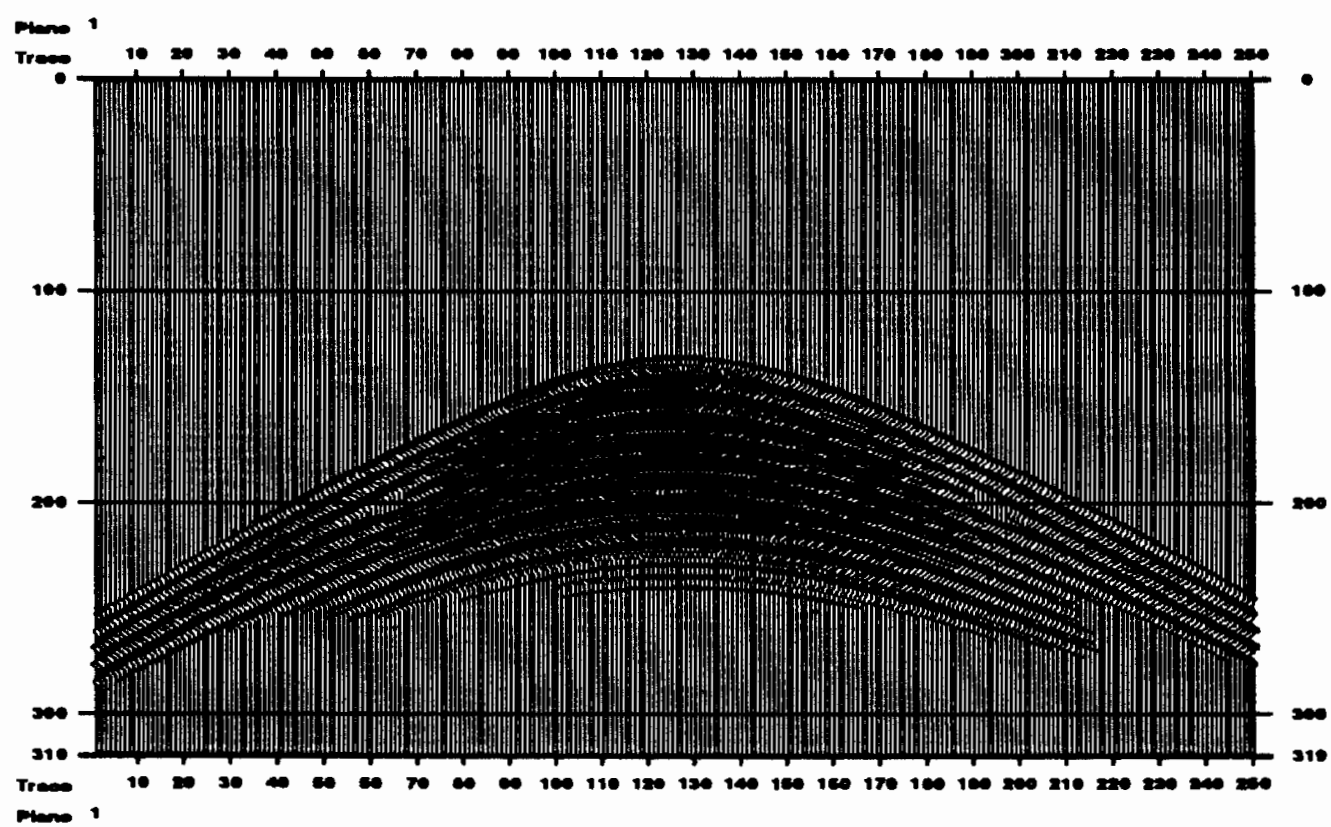

Figure 5.4: $\mathcal{F}_{j} \delta c_{j}-$ Pk Freq. $126 \mathrm{~Hz}$; No Lens. Horizontal axis $=x_{r}$, Vertical axis $=$ time $t_{r}$. 


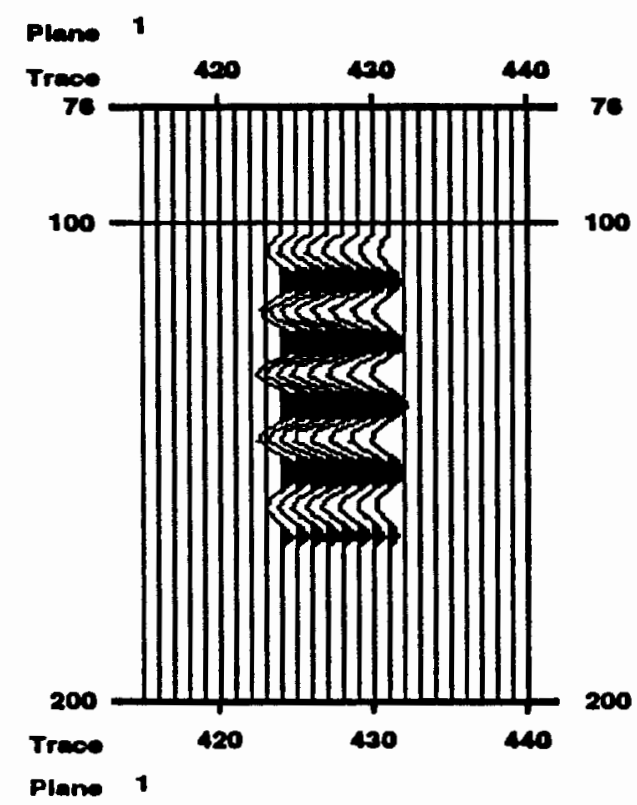

Figure 5.5: Reflectivity $\delta c_{j}$ - Pk Freq. $63 \mathrm{~Hz}$; Lens; $R_{j}=0.49 \mathrm{e}-3$. Horizontal axis $=\mathrm{x}$, vertical axis $=y$.

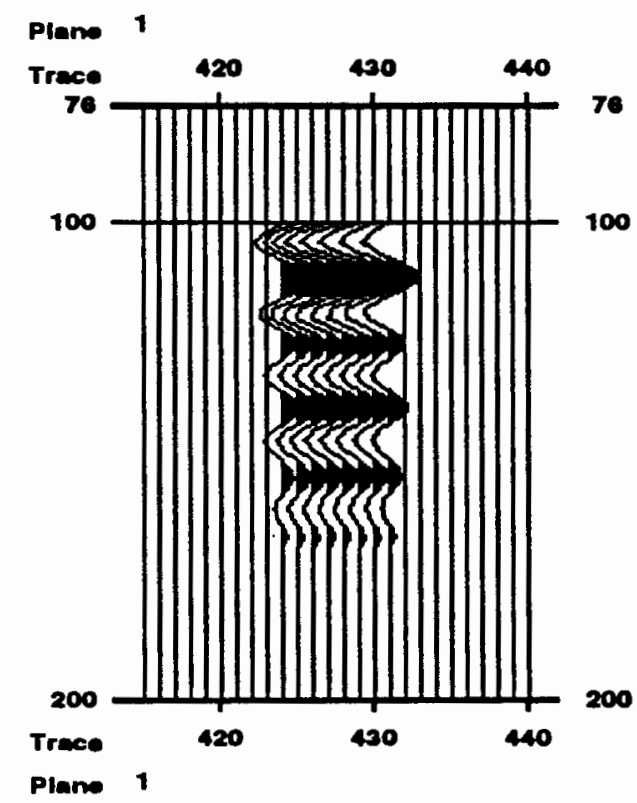

Figure 5.6: Reflectivity $\delta c_{j}$ - Pk Freq. $63 \mathrm{~Hz}$; No Lens; $R_{j}=0.99 \mathrm{e}-4$. Horizontal axis $=$ $\mathbf{x}$, vertical axis $=\mathbf{y}$. 


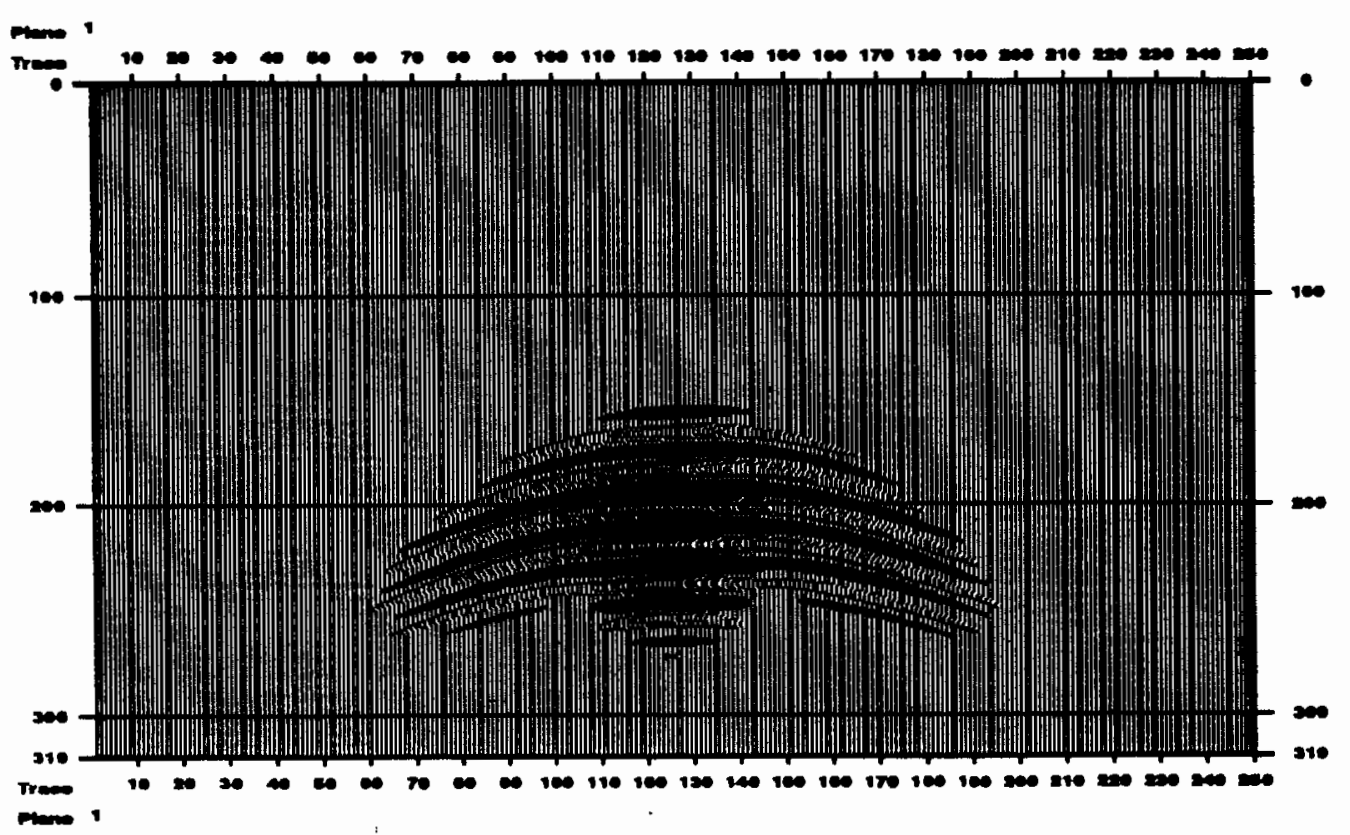

Figure 5.7: $\mathcal{F}_{j} \delta c_{j}-\mathrm{Pk}$ Freq. $63 \mathrm{~Hz}$; Lens. Horizontal axis $=x_{r}$, Vertical axis $=$ time $t_{r}$.

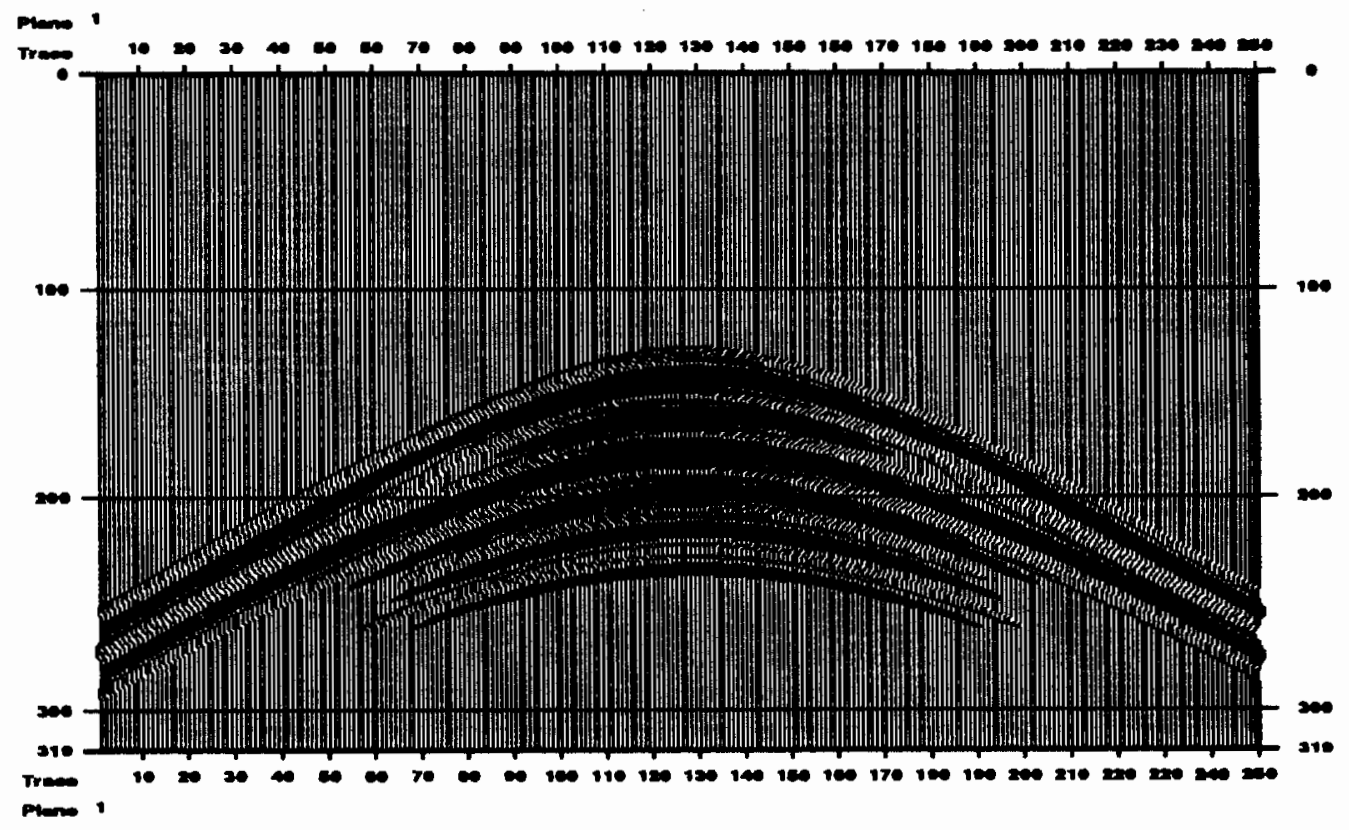

Figure 5.8: $\mathcal{F}_{j} \delta c_{j}$ - Pk Freq. $63 \mathrm{~Hz}$; No Lens. Horizontal axis $=x_{r}$, Vertical axis $=$ time $t_{\tau}$. 


\section{Figure 8.9}

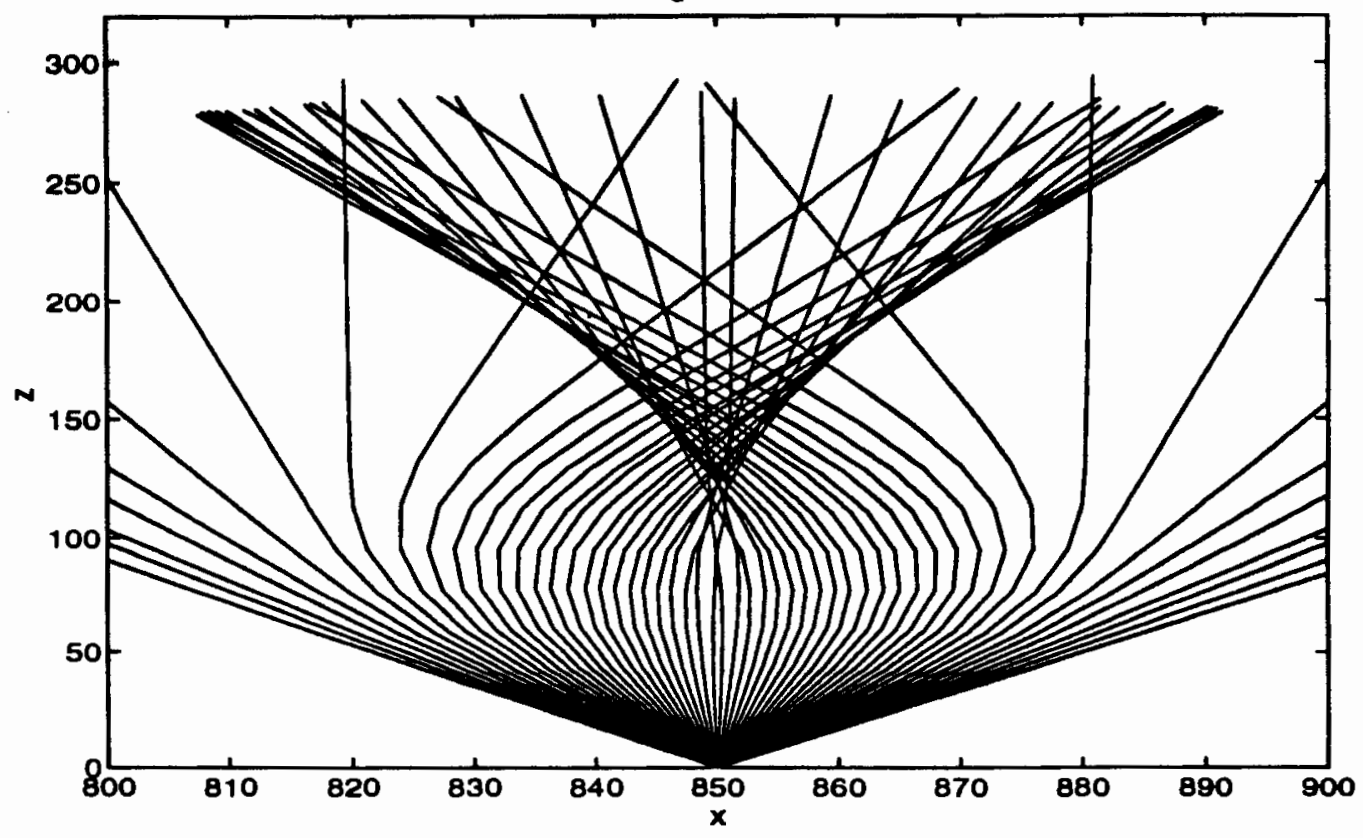

Figure 5.9: A picture of rays traced true the background medium $c_{0}$ with a lens. Note the cusped envelope $=$ a caustic curve.

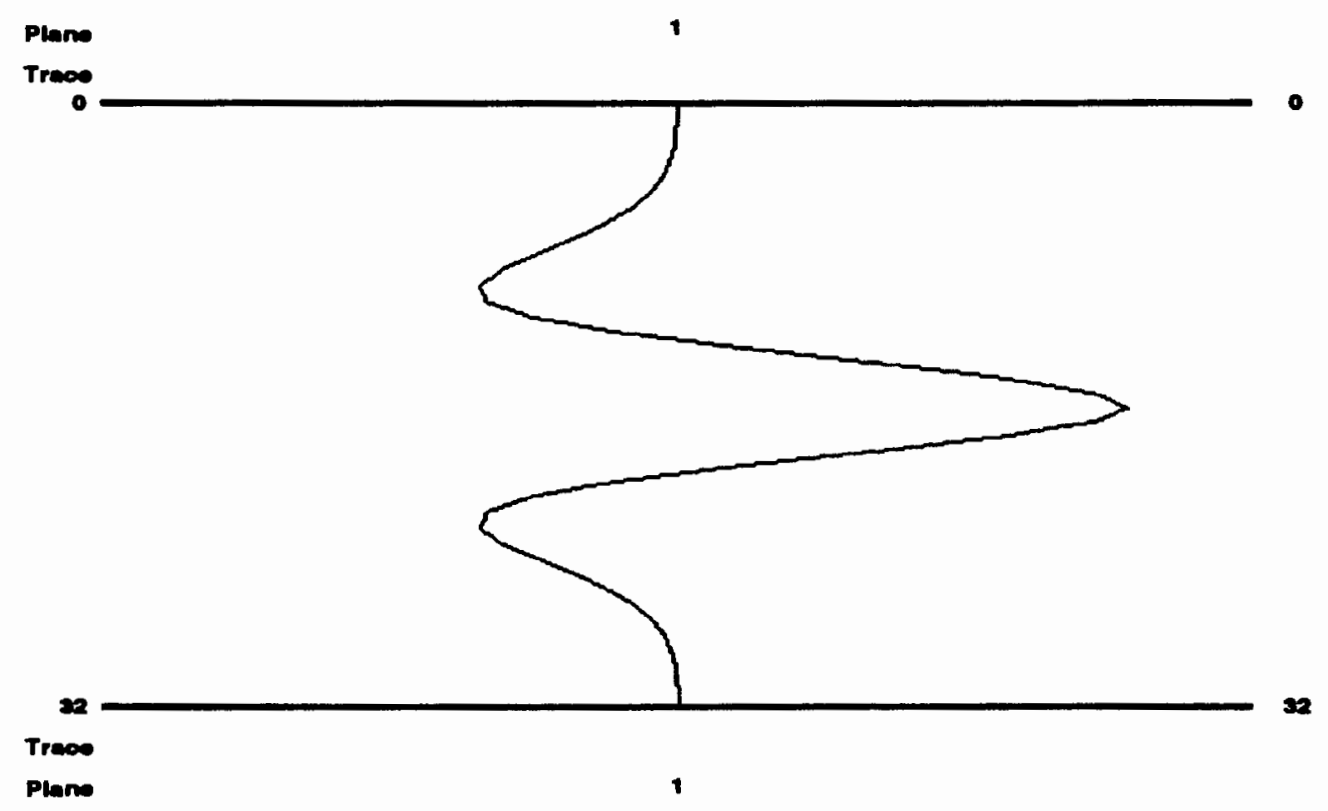

Figure 5.10: Ricker wavelet with $\omega=3.9 \mathrm{e}$. Horizontal axis = amplitude in meters, vertical axis $=$ time in milliseconds. 


\section{Conclusions}

We have shown that the forward map associated to the Linearized Wave equation is discontinuous in the prescence of caustics. This has important consequences for Linearized Inversion techniques. Any local methods, for example gradient based algorithms, will have obvious difficulties because of the loss of differentiability and/or continuity.

As a consequence of the unboundedness of $\mathcal{F}$ we have that the normal map cannot always be a Pseudodifferential Operator because if it were it would have an order $\mathrm{m}$ associated to it and this would mean that it would map $H_{c}^{s}\left(\Omega_{j}\right) \rightarrow H_{L o c}^{s-m}\left(\Omega_{j}\right)$ continuously but as we have just seen the operator grows without bound as we let $\Omega_{j}$ become closer to the caustic (for a sufficiently high frequency of incident waves).

The reader will notice that we needed to use a reflectivity field with indefinitely high frequency content in order to prove unboundedness. This is really the reason for the title of the paper; we can get lower estimates on the forward operator bound which grow without bound as we let $\Omega_{j}$ approach a caustic (for a sufficiently high frequency content in the reflectivity field). This means that we will see an "anomalous" reflection, meaning that we will see a large signal relative to the size of the reflectivity that reflected it back to the surface.

Note that the results in this paper, were for a two dimensional medium, but corresponding results hold in any dimension.

Finally the current numerical results are consistent with the theory. The extent to which this phenomenon may be observed is still undetermined at the moment. Further tests will be carried out in order to try to settle this question.

\section{Appendix}

\section{Existence of Plane wave reflectors}

In this section we will establish the intuitively obvious fact that the plane wave reflectors mentioned in the introduction do actually exist from a rigorous point of view.

Consider the setup depicted in figure (7.1). First we set down some notation. Let us denote by $X$ the two dimensional vector $(x, y)$ as indicated in the diagram. Let $V$ be a $C^{0}$ vector field which evaluates the unit vector parallel to the velocity vector of a ray leaving the source and passing through the point of evaluation (see diagram for example).

Now specify the direction in which you want the plane wave to be reflected i.e. specify a vector $r=\left(r_{1}, r_{2}\right)$; this choice will have to be restricted a little later but is more or less 
arbitrary in general. Then let $N_{0} \in T_{\left(x_{0}, y_{0}\right)} \mathrm{R}^{2}$ be of the form

$$
N_{0}=N\left(X_{0}\right)=N\left(x_{0}, y_{0}\right)=\frac{1}{\sqrt{1+\xi_{0}^{2}}}\left(-\xi_{0}, 1\right)
$$

(The reason of this choice is that eventually $N$ will be the normal vector of the graph generated by $y=\psi(x)$; for some $C^{1}$ function $\psi$.) Also choose $N$ in such a way that the following equation which expresses "Snell's Law of Reflection" is satisfied

$$
N_{0} \cdot\left(r+V\left(X_{0}\right)\right)=0
$$

thus we have that

$$
-r_{1} \xi_{0}-V_{1}\left(X_{0}\right) \xi_{0}+r_{2}+V_{2}\left(X_{0}\right)=0
$$

In general we want this latter equation to hold with $X_{0}$ replaced by $X$ and $\xi_{0}$ by $\xi(x)$ i.e. suppose we can have some reflecting curve given by the graph of $y=\psi(x)$, then the unit normal vector to it is given by

$$
\frac{1}{\sqrt{1+\xi^{2}(x)}}(-\xi(x), 1)
$$

where $\xi(x)=\nabla \psi(x)$. Therefore our task is complete if we can solve (locally) the equation

$$
F(x, \xi)=-r_{1} \xi-V_{1}(x, y(x)) \xi+r_{2}+V_{2}(x, y(x))=0
$$

(with $\xi=\xi(x)$ ) since then all we have to do is find the function $\psi$ by solving the ordinary differential equation

$$
\left\{\begin{array}{l}
\nabla \psi(x)=-\xi(x) \\
\psi\left(x_{0}\right)=y_{0}
\end{array}\right.
$$
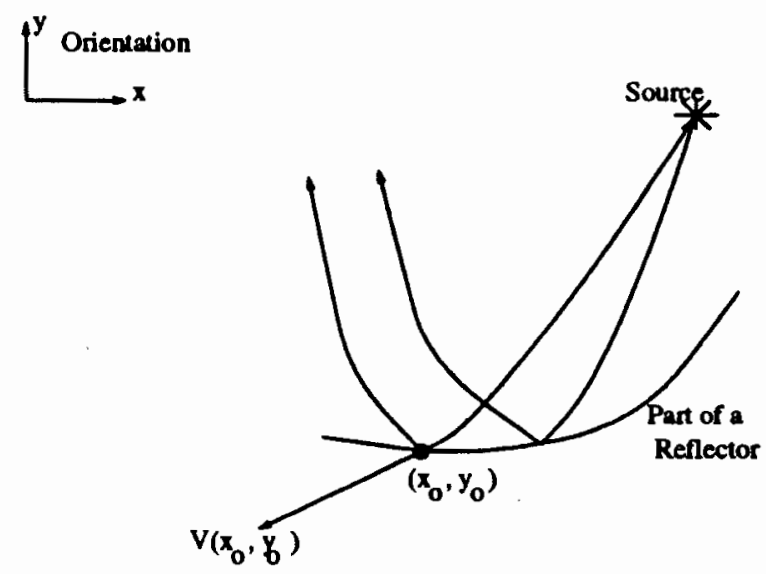

Figure 7.1: A Plane wave reflector

The question of local solvability of the equation (7.2) is answered by the Implicit Mapping Theorem which answers "yes" to the local existence of a $C^{0}$ function $\xi(x)$ satisfying 
equation (7.2) provided that $\frac{\partial F}{\partial \xi}\left(X_{0}, \xi_{0}\right) \neq 0$ i.e. $r_{1}+V_{1}\left(X_{0}\right) \neq 0$. Thus the choice of $r_{1}$ is restricted in a trivial way. This completes the proof of local existence of plane wave reflectors.

Acknowlegement: This work was partially supported by the National Science Foundation under research grant F49620-95-1-0039, the Office of Naval Research under research grant N00014-89-J-1115, the Texas Geophysical Parallel Computation Project, the Schlumberger Foundation, IBM, and The Rice Inversion Project. TRIP Sponsors for 1995 are Advance Geophysical, Amerada Hess, Amoco Production Co., Conoco Inc., Cray Research Inc., Discovery Bay, Exxon Production Research Co., Interactive Network Technologies, Mobil Research and Development Corp., and Texaco Inc.

\section{References}

[1] CHERYL B. BOSMAN PERCELL. "The effects of Caustics in Acoustic Inverse Scattering Experiments.” Ph.D. Thesis 1989, Department of Mathematical Sciences, Rice University, Houston, Tx 77005, TR89-3.

[2] R. COURANT and D. HILBERT. "Methods of Mathematical Physics", Vol. II (1962), published by Wiley.

[3] J.J. DUISTERMAAT. "Fourier Integral Operators" (1971), Lecture Notes, Courant Institute of Mathematical Sciences.

[4] K. HOPCRAFT, P. SMITH, "An introduction to electromagnetic inverse scattering." Kluwer Academic Press 1992.

[5] D. LUDWIG. "Uniform asymptotic expansions at a caustic." Comm. in pure and App. Math, Vol XIX, (1966).

[6] RAKESH. "A linearized inverse problem for the wave equation." In Partial Differential Equations, 13(5), 573-601 (1988).

[7] X. S. RAYMOND. "Elementary Introduction to the Theory of Pseudodifferential Operators (1991)." Studies in Advanced Mathematics, CRC Press.

[8] W. W. SYMES. “Mathematical Foundations of Reflected Wave Imaging." Department of Mathematical Sciences, Rice University, Houston, Tx 77005, TR90-2 (1990).

[9] M. E. TAYLOR. "Pseudodifferential Operators." Princeton University Press 1981.

[10] A.P.E. TEN KROODE, D.-J. SMIT, A.R. VERDEL. "Linearized Inverse Scattering In The Presence Of Caustics." SPIE-Conference On Math. Methods In Geophysical Imaging, San Diego, July 24-29 1994.

[11] B. White. "The stochastic caustic." SIAM J. Appl. Math., 44:127-149, 1982. 
[12] F. TREVES. "Introduction to Pseudodifferential Operators and Fourier Integral Operators. Vols $I$ and II." The University Series in Mathematics. Plenum Press, New York.

[13] L. HORMANDER. “Fourier Integral Operators I." Acta Math. 127 (1971).

[14] J.J. DUISTERMAAT and L. HORMANDER. Fourier Integral Operators II." Acta Math. 128 (1972).

[15] V. P. MASLOV and M. V. FEDORIUK. "Semi-Classical approximation in quantum mechanics." D. Reidel publishing co., 1981.

[16] L. HORMANDER. “Linear Partial Differential Operators I." Springer Verlag, 1990.

[17] J.J. DUISTERMAAT. "Oscillatory Integrals, Lagrange Immersions, and unfoldings of Singularities." Comm. Pure and App. Math., Vol. XXVII, p.207 - 281 (1974). 
